\title{
Dynamic Characterization and Stabilization of Large Gravity-Tractor Designs
}

\author{
Eugene G. Fahnestock* and Daniel J. Scheeres \\ University of Michigan, Ann Arbor, Michigan 48109
}

DOI: $10.2514 / 1.32554$

\begin{abstract}
This paper addresses the problem of stabilizing and regulating, to a desired reference, the attitude and position of large gravity-tractor spacecraft relative to near-Earth objects to be deflected from Earth impact. We analyze gravity tractors constructed in both the previously proposed pendulum configuration and a novel bar configuration. These interact with the deflection target through mutual gravitational potential, such that the system has fully coupled rotational and translational dynamics. The target body is represented by increasingly more realistic models (sphere, oblate spheroid, general triaxial ellipsoid, and polyhedral mesh). For the simplest of these, an eigenstructure-based controller is detailed, followed by an energy-based controller for the more general target representations, with additional dissipative feedback used in each case. It is demonstrated with theory and simulation results that both the pendulum and novel bar gravity tractors are suitably controlled to the most desirable precessing downtrack-towingdirection vector using these controller designs. They are robust in the presence of realistic perturbations and the effects of full geometric detail for both the natural and artificial bodies. Further, this paper introduces important performance metrics for gravity-tractor system design, which quantify the difficulty of control for each case. Based on this, some prior claims made to the effect that because the gravity tractor does not need to physically connect with the asteroid, there is no need for prior knowledge of the asteroid's shape, composition, rotation rate, etc., appear to be incorrect.
\end{abstract}

\section{Introduction}

$\mathbf{T}$ HE so-called gravity tug or gravity tractor (GT) has recently been proposed as a system for altering the heliocentric orbital velocity of a near-Earth object (NEO) to prevent it from impacting Earth. The GT concept, originally presented in [1] and further discussed in [2], places a spacecraft in close proximity to the NEO, hovering with respect to the NEO center of mass by using thrust from continuously operating, high- $I_{\mathrm{sp}}$ electric propulsion to balance the gravitational force exerted on the spacecraft by the NEO. If the thruster exhaust avoids impinging on the NEO surface, the net result is a continuous acceleration of the combined NEO and spacecraft system. Such a towing action in the direction of net thrust on the whole system will, over an extended operation time, produce the small velocity change $\Delta V$ needed to avoid impact. If the operation is conducted 10-20 years before impact, the $\Delta V$ required is typically less than $1 \mathrm{~cm} / \mathrm{s}$ [3] , and this may be further reduced by a few orders of magnitude if the NEO's projected impact is preceded by a resonant-return Earth flyby.

The GT has many advantages, including 1) no use of nuclear detonations or kinetic impacts with highly uncertain outcomes, 2) no requirement for attachment or anchoring to the NEO surface as in [4], 3 ) no thruster gimballing or on-off operation, with heavy power cycling needed to thrust only in approximately the optimal direction, and 4) continuous high-accuracy monitoring of the NEO trajectory and remaining impact probability, both initially and throughout the deflection operation. Disadvantages include the extended operation time and long technology development and construction times, though the latter are reduced for a smaller (in mass and size) GT that

Presented as Paper P2-5 at the 2007 Planetary Defense Conference: Protecting Earth from Asteroids, Washington, D.C., 5-8 March 2007; received 2 June 2007; accepted for publication 28 August 2007. Copyright $($ ) 2007 by the American Institute of Aeronautics and Astronautics, Inc. All rights reserved. Copies of this paper may be made for personal or internal use, on condition that the copier pay the $\$ 10.00$ per-copy fee to the Copyright Clearance Center, Inc., 222 Rosewood Drive, Danvers, MA 01923; include the code 0731-5090/08 \$10.00 in correspondence with the CCC.

${ }^{*}$ Graduate Student, Department of Aerospace Engineering, 2008 FXB Building, 1320 Beal Avenue. Student Member AIAA.

${ }^{\dagger}$ Associate Professor, Department of Aerospace Engineering, 3048 FXB Building, 1320 Beal Avenue. Associate Fellow AIAA. does not require currently unavailable longer-lifetime and higherthrust electric propulsion devices together with nuclear electric power systems. Regardless, long warning times before projected impact are necessary for use of the GT.

An additional (and, as yet, unaddressed in literature) disadvantage applies to a large GT. Here, large means having spatial dimensions that are an appreciable fraction of the mean radius of the NEO to be deflected, and it usually also means having mass that is large relative to interplanetary spacecraft built to date. The GT mass is still very much smaller than that of the NEO, but it is the significant spatial distribution of that GT mass that gives rise to this additional disadvantage, first addressed herein. This is the difficulty of operating the GT in a controlled manner, given its complex dynamic interaction with the NEO body, characterized by fully coupled translational and rotational dynamics. All previous treatments of the GT concept do not give any serious consideration to this coupled behavior. These all show a pendulum configuration, with the majority of the mass of the GT hanging below outboard canted thrusters at the tip of a boom extending away from the NEO along the local vertical (see Fig. 1). The familiar gravity-gradient stability of satellites orbiting what is a nearly spherical, and comparatively large in dimension, planetary body is assumed to translate to stability of the pendulum GT hovering under a nominal thrust level next to a potentially very nonspherical, and comparatively similar in dimension, planetesimal. Dynamic intuition suggests that this may not hold true, as mentioned in [ㅁ] and explained in [6]

Herein, we introduce a series of analytical models for the coupled system in which the NEO is represented using progressively fewer simplifying assumptions (first as a sphere and then as an oblate spheroid or general triaxial ellipsoid). For each NEO representation, the GT is represented both in the pendulum configuration and in a bar configuration. The latter has a main body and two thruster nodes, each at the end of a long, lightweight boom extending horizontally with respect to the local vertical (see Fig. 2). Our motivation behind examining the new bar configuration is the hypothesis that it allows better placement and orientation of thrusters, still consistent with avoiding exhaust plume impingement, to achieve a reduced ratio of engine thrust required to net towing effect. For mutual gravity computation, we represent the pendulum GT by two rigidly linked point masses, one at the main body and one at the thruster node, and as an ellipsoid (as illustrated in Fig. 1). We represent the bar GT by 


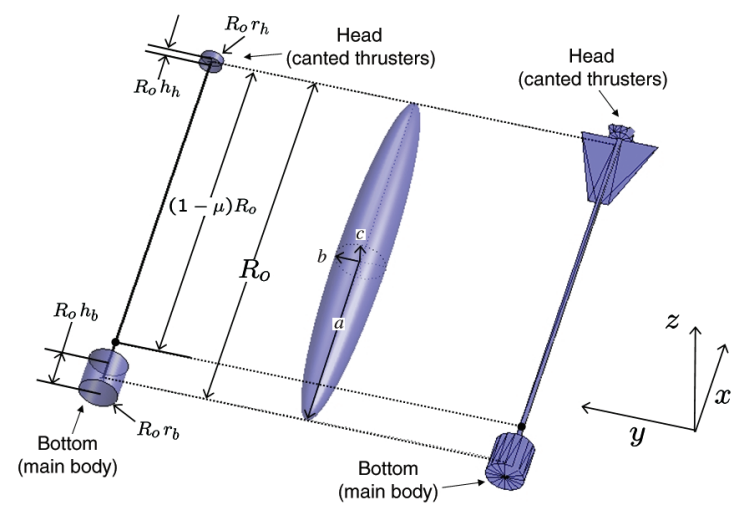

Fig. 1 Illustration of point-mass (left), ellipsoid (center), and detailed polyhedral (right) models for the pendulum GT configuration. The GTfixed frame orientation shown has its origin at the center of mass in each case.

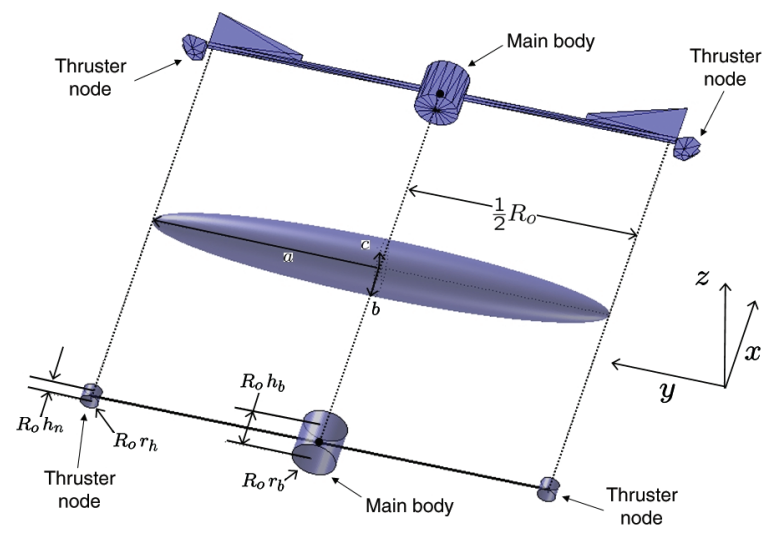

Fig. 2 Illustration of point-mass (bottom), ellipsoid (center), and detailed polyhedral (top) models for the bar GT configuration. The GTfixed frame orientation shown has its origin at the center of mass in each case.

three rigidly linked point masses and a differently oriented ellipsoid (as illustrated in Fig. 2). Though grossly simplified, this whole series of analytical models builds up to a very nontrivial system, sufficient for development of a controller that performs suitably well when tested within a no-simplifications numerical validation model. In that model, both the NEO and the GT are represented as detailed polyhedral shapes with internal mass distribution.

The paper is organized as follows: In Sec. II, for every model with a spherical NEO representation, the model's equations are presented, along with the nominal control force and moment required to act on the GT to enforce desired relative equilibria. Variational methods are used to obtain equations for the perturbation dynamics about those desired equilibria. We analyze the stability of the new linear equations for each model to characterize the overall system's behavior in the presence of small perturbations arising from the many features really present and not included in the clean analytical model. We devise eigenstructure-based control laws and augment them with additional rate feedback to give control forces and moments to add to the nominal ones, to produce stability in the face of these perturbations. In Sec. III, for every model with more general NEO representations, we present the full system Hamiltonian and obtain much simplified reduced equations of motion using valid assumptions. We present a potential-shaping or energy-based derivation of control forces and moments, and also augment them with the same nonconservative rate feedbacks obtained earlier. In Sec. IV, we examine four specific scenarios for deflecting two target bodies in the current set of known asteroids with nonzero probability of Earth impact, using both pendulum and bar GTs for each. Best guesses are made for unknown target-body properties. For each of these scenarios, closed-loop simulation results from propagating the full nonlinear analytical model and from propagating the "truth" numerical validation model, with polyhedral representations of both the NEO and GT, are shown together and the results are compared. Section $\underline{\mathrm{V}}$ provides a discussion (and quantification for each test scenario) of the performance penalties incurred by using the appropriate control scheme. We discuss the implications of the performance decreases, the level of difficulty of controlling the whole system, and the need for characterizing deflection targets before a GT mission is designed. We also discuss the favorability (or lack thereof) of choosing the novel bar configuration over the previously proposed pendulum configuration, and we offer conclusions in Sec. VI.

\section{Homogenous Sphere Representation of NEO}

The simplest case to begin with is to represent the NEO as a uniform-density spherical body. This temporarily allows for decoupling of the rotational dynamics of the asteroid from the rotational dynamics of the GT and from all translational dynamics. In fact, the attitude of the GT itself is decoupled from the dynamics of the internal variables of relative position, relative velocity, and the angular velocity of the GT. Following the development in [6], we have the following equations written with respect to the body frame fixed in the GT:

$$
\ddot{\mathbf{R}}+\dot{\boldsymbol{\Omega}}_{1} \times \mathbf{R}+2 \boldsymbol{\Omega}_{1} \times \dot{\mathbf{R}}+\boldsymbol{\Omega}_{1} \times \boldsymbol{\Omega}_{1} \times \mathbf{R}=-\frac{\mathbf{F}_{1}}{m}-\frac{\mathbf{F}_{c}}{m_{1}}
$$

$$
I_{1} \dot{\boldsymbol{\Omega}}_{1}+\boldsymbol{\Omega}_{1} \times I_{1} \boldsymbol{\Omega}_{1}=\mathbf{M}_{1}+\mathbf{M}_{c}
$$

where the subscripts 1 and 2 refer to the GT and the NEO, respectively, and we have adopted the convention of writing vectors in bold type and their magnitudes or other scalars in regular type. Matrices and dyads are also in regular type, but they should be easy to identify as such. Let $\mathbf{R}$ be the relative position vector from the GT's center of mass to the NEO's center of mass, let $\Omega$ be an angular velocity with respect to an inertial frame, and let $I$ be an inertia dyad. $\mathbf{F}_{1}$ and $\mathbf{M}_{1}$ are the combined force and moment acting on the GT due to mutual gravity, and $\mathbf{F}_{c}$ and $\mathbf{M}_{c}$ are the sums of all effective control forces and moments due to thrusters and/or momentum wheels. By effective, we mean acting through and about the center of mass of the GT, as distinguished from actual thruster forces and wheel moments. Here, the mass parameter for the system is determined from the mass of the GT, $m_{1}$, and the mass of the NEO, $m_{2}$, via

$$
m=\frac{m_{1} m_{2}}{m_{1}+m_{2}}
$$

Also, for the spherical NEO model, the force and moment due to mutual gravity are

$$
\mathbf{F}_{1}=-\mathbf{F}_{2}=-\frac{\partial U}{\partial \mathbf{R}}, \quad \mathbf{M}_{1}=\mathbf{R} \times\left(-\frac{\partial U}{\partial \mathbf{R}}\right)
$$

where the mutual gravity force potential is given by

$$
U=G m_{2} \int_{1} \frac{\mathrm{d} m(\rho)}{\|\mathbf{R}-\boldsymbol{\rho}\|}
$$

where $\rho$ is the vector from the center of mass of the GT to each differential mass element in the GT. If we let $\tilde{\mathbf{a}}$ denote the skewsymmetric cross-product dyad for vector a and we normalize all quantities by a representative length $R_{o}$, mass, and time, we obtain

$$
\begin{gathered}
\mathbf{r}^{\prime \prime}+\widetilde{\omega_{1}^{\prime}} \mathbf{r}+2 \widetilde{\omega_{1}} \mathbf{r}^{\prime}+\widetilde{\omega_{1}} \widetilde{\omega_{1}} \mathbf{r}=-\mathbf{f}_{1}-\mathbf{f}_{c} \\
I_{1} \omega_{1}^{\prime}+\widetilde{\omega_{1}} I_{1} \omega_{1}=\mathbf{m}_{1}+\mathbf{m}_{c}
\end{gathered}
$$

where the prime denotes differentiation with respect to the new timescale and we made use of the following conversions: 


$$
\begin{array}{cl}
\mathbf{r}=\frac{\mathbf{R}}{R_{o}}, \quad \overline{\boldsymbol{\rho}}=\frac{\rho}{R_{o}}, & \boldsymbol{\omega}_{1}=\frac{\mathbf{\Omega}_{1}}{n}, \quad I_{1}=\frac{\mathrm{I}_{1}}{m_{1} R_{o}^{2}} \\
\mathbf{f}_{c}=\frac{\mathbf{F}_{c}}{m_{1} n^{2} R_{o}}, \quad \mathbf{m}_{c}=\frac{\mathbf{M}_{c}}{m_{1} n^{2} R_{o}^{2}} &
\end{array}
$$

where $n$ is the mean motion of the system, from $n=\sqrt{G\left(m_{1}+m_{2}\right) / R_{o}^{3}}$. Also, the normalized versions of the mutual gravity force and moment on the GT and of the underlying mutual potential are now

$$
\mathbf{f}_{1}=-\frac{\partial u}{\partial \mathbf{r}}, \quad \mathbf{m}_{1}=v \tilde{\mathbf{r}}\left(-\frac{\partial u}{\partial \mathbf{r}}\right), \quad u=\frac{1}{m_{1}} \int_{1} \frac{\mathrm{d} m(\bar{\rho})}{\|\mathbf{r}-\bar{\rho}\|}
$$

where the unitless parameter $v=m_{2} /\left(m_{1}+m_{2}\right)$ is like that commonly used in the restricted three-body problem.

$$
\begin{aligned}
& \delta \mathbf{r}^{\prime \prime}+\widetilde{\delta \omega_{1}^{\prime}} \mathbf{r}+\widetilde{\omega_{1}^{\prime}} \delta \mathbf{r}+2 \widetilde{\delta \omega_{1}} \mathbf{r}^{\prime}+2 \widetilde{\omega_{1}} \delta \mathbf{r}^{\prime}+\widetilde{\delta \omega_{1}} \widetilde{\omega_{1}} \mathbf{r} \\
& +\widetilde{\omega_{1}} \widetilde{\delta \omega_{1}} \mathbf{r}+\widetilde{\omega_{1}} \widetilde{\omega_{1}} \delta \mathbf{r}=\frac{\partial^{2} u}{\partial \mathbf{r}^{2}} \delta \mathbf{r}-\delta \mathbf{f}_{c} \\
& I_{1} \delta \boldsymbol{\omega}_{1}^{\prime}+\widetilde{\delta \omega_{1}} I_{1} \omega_{1}+\widetilde{\omega_{1}} I_{1} \delta \boldsymbol{\omega}_{1}=-v \widetilde{\delta} \mathbf{r} \frac{\partial u}{\partial r}-v \tilde{\mathbf{r}} \frac{\partial^{2} u}{\partial r^{2}} \delta \mathbf{r}+\delta \mathbf{m}_{c}
\end{aligned}
$$

These can be combined into the following matrix form:

$$
\left[\begin{array}{c}
\delta \mathbf{r}^{\prime} \\
\delta \mathbf{r}^{\prime \prime} \\
\delta \boldsymbol{\omega}_{1}^{\prime}
\end{array}\right]=A\left[\begin{array}{c}
\delta \mathbf{r} \\
\delta \mathbf{r}^{\prime} \\
\delta \boldsymbol{\omega}_{1}
\end{array}\right]+B\left[\begin{array}{c}
\delta \mathbf{f}_{c} \\
\delta \mathbf{m}_{c}
\end{array}\right]
$$

with

$$
A=\left[\begin{array}{ccc}
0_{3 \times 3} & U_{3 \times 3} & 0_{3 \times 3} \\
-\widetilde{\boldsymbol{\omega}_{1}} \widetilde{\boldsymbol{\omega}_{1}}+\frac{\partial^{2} u}{\partial \mathbf{r}^{2}}+\nu \tilde{\mathbf{r}} I_{1}^{-1}\left[\frac{\partial u}{\partial \mathbf{r}}-\tilde{\mathbf{r}} \frac{\partial^{2} u}{\partial \mathbf{r}^{2}}\right] & -2 \widetilde{\omega_{1}} & \widetilde{\omega_{1}} \tilde{\mathbf{r}}+\left(\widetilde{\boldsymbol{\omega}_{1} \mathbf{r}}\right)+\tilde{\mathbf{r}} I_{1}^{-1}\left[\widetilde{I_{1} \boldsymbol{\omega}_{1}}-\widetilde{\boldsymbol{\omega}_{1}} I_{1}\right] \\
\nu I_{1}^{-1}\left[\frac{\widetilde{\partial u}}{\partial \mathbf{r}}-\tilde{\mathbf{r}} \frac{\partial^{2} u}{\partial \mathbf{r}^{2}}\right] & 0_{3 \times 3} & I_{1}^{-1}\left[\widetilde{I_{1} \boldsymbol{\omega}_{1}}-\widetilde{\boldsymbol{\omega}_{1}} I_{1}\right]
\end{array}\right], \quad B=\left[\begin{array}{cc}
0_{3 \times 3} & 0_{3 \times 3} \\
-U_{3 \times 3} & \tilde{\mathbf{r}} I_{1}^{-1} \\
0_{3 \times 3} & I_{1}^{-1}
\end{array}\right]
$$

With Eqs. (ㅁ) and (ㅁ), we determine the nominal control force $\mathbf{f}_{c_{\mathrm{eq}}}$ and moment $\overline{\mathbf{m}}_{c_{\mathrm{eq}}}$ needed to enforce desired relative equilibria and then take the variation of the equations of motion about those desired equilibria, to obtain (in this case, linear) perturbation-dynamics systems. The control laws to drive these systems to the origin or keep them bounded determine additional $\delta \mathbf{f}_{c}$ and $\delta \mathbf{m}_{c}$ to be added to the nominal control force and moment to yield the total control force and moment. Herein, we examine two desired equilibria. For the first and more general one, the GT is rotating with respect to inertial space and translating relative to the NEO, so that the towing direction remains parallel or antiparallel to the NEO's heliocentric orbit velocity vector. This is the optimal alignment for adding or removing energy from its orbit about the sun and thereby reducing impact risk, according to previous studies $[\underline{4}, 7]$. We can assume, without loss of generality, an addition of energy to the heliocentric orbit. This may be referred to as towing in the downtrack direction. Given an approximately circular NEO orbit, that orbit's velocity vector rotates at the roughly constant rate equal to the NEO's normalized mean motion about the sun, $n_{\text {sun }}$. The second equilibrium involves the GT not rotating with respect to inertial space, so that the towing direction is fixed in the inertial frame, and this always falls out as a special case of the first equilibrium. For the first equilibrium, we find

$$
\mathbf{f}_{c_{\mathrm{eq}}}=-\widetilde{\boldsymbol{\omega}_{1}} \widetilde{\boldsymbol{\omega}_{1}} \mathbf{r}+\frac{\partial u}{\partial \mathbf{r}}, \quad \mathbf{m}_{c_{\mathrm{eq}}}=\widetilde{\boldsymbol{\omega}_{1}} I_{1} \boldsymbol{\omega}_{1}+v \tilde{\mathbf{r}} \frac{\partial u}{\partial r}
$$

and $\omega_{1}$ is just replaced by zero for the second equilibrium. Taking the full variations of Eqs. (ㅁ) and (ㅁ), we have where $U_{3 \times 3}$ denotes the identity dyad, and $0_{3 \times 3}$ denotes a $3 \times 3$ block of zero elements. This linear matrix equation for perturbation dynamics is used as written for the first equilibrium, with the rotation of the GT to preserve the optimal towing direction. For the second equilibrium, we again replace all instances of $\omega_{1}$ with zero, leading to great simplification. Because conservation of total angular momentum and energy no longer apply, due to the action of the GT thrusters, the usual constraints due to those usually conserved integrals do not apply, such that we cannot further reduce the dimension of the plant matrix in Eq. (12).

\section{A. Pendulum Configuration}

With this configuration, the GT has two parts separated by a boom: a bottom, containing the power plant, etc., and a head, to which the main thrusters are mounted. We choose to align the unit vectors $(\hat{\mathbf{i}}, \hat{\mathbf{j}}, \hat{\mathbf{k}})$ for the frame fixed to the GT so that $\hat{\mathbf{i}}$ points from the GT centroid along the boom through the head. Then, in the basis of these unit vectors, at either of the desired equilibria, the vectors $\mathbf{r}$ and $\partial u / \partial \mathbf{r}$ both have components only in the $\hat{\mathbf{i}}$ direction, denoted as $x$ and $u_{x}$, respectively. Assuming symmetry of the GT about the $X-Y$ and $X-Z$ planes, a reasonable assumption, the matrix $\partial^{2} u / \partial \mathbf{r}^{2}$ will also have only diagonal elements, denoted as $u_{x x}, u_{y y}$, and $u_{z z}$ respectively. In terms of these scalar components, for the first desired equilibrium, the nominal control force and moment are obtained from Eqs. (9) as $\mathbf{f}_{c_{\mathrm{eq}}}=\left(n_{\mathrm{sun}}^{2} x+u_{x}\right) \hat{\mathbf{i}}$ and $\mathbf{m}_{c_{\mathrm{eq}}}=0$, and the plant and input matrices for the perturbation dynamics are obtained from Eq. (12) as

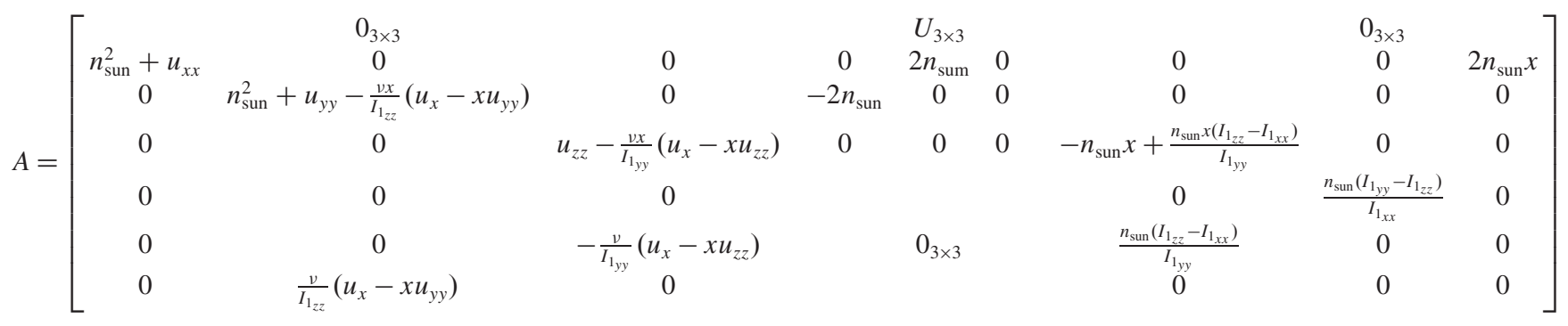




$$
B=\left[\begin{array}{cccc}
0_{3 \times 3} & & 0_{3 \times 3} & \\
& 0 & 0 & 0 \\
-U_{3 \times 3} & 0 & 0 & -x / I_{1_{z z}} \\
& 0 & x / I_{1_{y y}} & 0 \\
& 1 / I_{1_{x x}} & 0 & 0 \\
0_{3 \times 3} & 0 & 1 / I_{1_{y y}} & 0 \\
& 0 & 0 & 1 / I_{1_{z z}}
\end{array}\right]
$$

For the second equilibrium with an inertially fixed towing direction, the plant matrix simplifies as we set $n_{\text {sun }}$ to zero, whereas the input matrix is the same. We notice that the system of these perturbation dynamics is fully controllable everywhere in the parameter space, regardless of which representation we use for the pendulum GT, which can be verified by the fact that the first nine columns of the controllability test matrix $\left[\begin{array}{llll}B & A B & \cdots & A^{8} B\end{array}\right]$ are

$$
\left[\begin{array}{ccccccc}
0_{3 \times 3} & & 0_{3 \times 3} & & & -U_{3 \times 3} & \\
& 0 & 0 & 0 & 0 & 2 n_{\text {sun }} & 0 \\
-U_{3 \times 3} & 0 & 0 & -x / I_{1_{z z}} & -2 n_{\text {sun }} & 0 & 0 \\
& 0 & x / I_{1_{y y}} & 0 & 0 & 0 & 0 \\
& 1 / I_{1_{x x}} & 0 & 0 & & & \\
0_{3 \times 3} & 0 & 1 / I_{1_{y y}} & 0 & & 0 & 0 \\
& 0 & 0 & 1 / I_{1_{z z}} & & &
\end{array}\right]
$$

which has full rank, as long as $I_{1_{x x}}$ is not allowed to approach zero. The rank does not change with setting $n_{\text {sun }}$ to zero, and so full controllability is maintained for the second equilibrium too.

Consider that second equilibrium, and notice that without any additional control moment other than the nominal one, the angular velocity perturbation dynamics are simply driven by the position perturbation (i.e., $\delta \boldsymbol{\omega}_{1}^{\prime}=S \delta \mathbf{r}$ ), with $S$ being a sparse matrix that becomes skew-symmetric if the pendulum GT is axisymmetric about the local vertical. Therefore, for the second equilibrium, it will be sufficient to determine whatever additional control force is needed so as to ensure the stability of the otherwise decoupled position and velocity perturbation dynamics, and the angular velocity perturbation dynamics will also be rendered stable. Looking at just the former dynamics, we can readily see from writing the matrix equations in second-order form that the eigenvalues will be given by

$$
\begin{gathered}
\pm \sigma_{x}= \pm \sqrt{u_{x x}}, \quad \pm \sigma_{y}= \pm \sqrt{u_{y y}-\frac{v x}{I_{1_{z z}}}\left(u_{x}-x u_{y y}\right)} \\
\pm \sigma_{z}= \pm \sqrt{u_{z z}-\frac{v x}{I_{1_{y y}}}\left(u_{x}-x u_{z z}\right)}
\end{gathered}
$$

The associated pairs of eigenvectors are given by

$$
\begin{aligned}
& \mathbf{V}_{ \pm x}=\left[\begin{array}{llllll}
\frac{1}{\sqrt{1+\sigma_{x}^{2}}} & 0 & 0 & \frac{ \pm \sigma_{x}}{\sqrt{1+\sigma_{x}^{2}}} & 0 & 0
\end{array}\right]^{T} \\
& \mathbf{V}_{ \pm y}=\left[\begin{array}{llllll}
0 \frac{1}{\sqrt{1+\sigma_{y}^{2}}} & 0 & 0 & \frac{ \pm \sigma_{y}}{\sqrt{1+\sigma_{y}^{2}}} & 0
\end{array}\right]^{T} \\
& \mathbf{V}_{ \pm z}=\left[\begin{array}{llllll}
0 & 0 \frac{1}{\sqrt{1+\sigma_{z}^{2}}} & 0 & 0 & \frac{ \pm \sigma_{z}}{\sqrt{1+\sigma_{z}^{2}}}
\end{array}\right]^{T}
\end{aligned}
$$

We adopt a simple feedback control approach, patterned after that used in [8] to stabilize naturally unstable periodic orbits. The key idea behind this approach is the use of a feedback law specified by the instantaneous eigenvalue and eigenvector structure of the system to produce stability in the sense of Lyapunov (not asymptotic) by removing (through pole placement) both the hyperbolic unstable and stable modes in matched pairs and leaving the other modes that are pure oscillatory untouched. Specifically, for every $\pm \sigma_{i}$ pair that is one positive real number and one negative real number, we use

$$
\begin{gathered}
\delta \mathbf{f}_{c_{i}}=\sigma_{i}^{2} g_{i}\left[\mathbf{V}_{+i_{(1: 3)}} \mathbf{V}_{+i_{(1: 3)}}^{T}+\mathbf{V}_{-i_{(1: 3)}} \mathbf{V}_{-i_{(1: 3)}}^{T}\right] \delta \mathbf{r} \\
g_{i}>1, \quad i \in\{x, y, z\}
\end{gathered}
$$

which is the square of the unstable mode's characteristic exponent, times a tunable scalar gain $g_{i}$, times the sum of outer products of the upper (position perturbation) half of the two eigenvectors matching with the eigenvalues of the stable and unstable modes, postmultiplied by the position perturbation itself. The total additional control force $\delta \mathbf{f}_{c}$ is the sum of the $\delta \mathbf{f}_{c_{i}}$ across all $i$.

For the first equilibrium, the eigenstructure of the plant matrix is not as convenient to determine. But the dynamics decouple between two sets evolving on two subspaces that we can separate from each other, one with five states ( $X$ and $Y$ components of position and velocity, plus the $Z$ component of angular velocity) and the other with four states ( $Z$ component of position and velocity, plus the $X$ and $Y$ components of angular velocity).

For the five-state subspace and the dynamics on it, one eigenvalue is zero and the characteristic exponents for the other modes are given by

$$
\begin{gathered}
\sigma_{x}^{2}, \sigma_{y}^{2}=-\frac{1}{2}\left[2 n_{\text {sun }}^{2}-u_{x x}-u_{y y}+\frac{v x}{I_{1 z z}}\left(u_{x}-x u_{y y}\right)\right] \pm \frac{1}{2}\left\{\left[2 n_{\text {sun }}^{2}\right.\right. \\
\left.-u_{x x}-u_{y y}+\frac{v x}{I_{1_{z z}}}\left(u_{x}-x u_{y y}\right)\right]^{2}-4\left[4 n_{\text {sun }}^{2} \frac{v x}{I_{1_{z z}}}\left(u_{x}-x u_{y y}\right)\right. \\
\left.\left.+\left(n_{\text {sun }}^{2}+u_{y y}-\frac{v x}{I_{1_{z z}}}\left(u_{x}-x u_{y y}\right)\right)\left(n_{\text {sun }}^{2}+u_{x x}\right)\right]\right\}^{\frac{1}{2}}
\end{gathered}
$$

With $n_{\text {sun }}=0$, elimination of terms in the preceding recovers the top two of Eqs. (15). Otherwise, for the right-hand side of the preceding to be either a positive real number (leading to the corresponding modes being hyperbolic stable and unstable) or negative real number (leading to the corresponding mode being an undamped oscillation), the preceding quantity in braces needs to be positive. Written out in detail, this condition is

$$
\begin{gathered}
\left(u_{x x}-u_{y y}\right)^{2}+\left(\frac{v x}{I_{1_{z z}}}\left(u_{x}-x u_{y y}\right)\right)^{2}+3 u_{x x} \frac{v x}{I_{1_{z z}}}\left(u_{x}-x u_{y y}\right) \\
-8 n_{\text {sun }}^{2}\left(u_{x x}+u_{y y}+\frac{v x}{I_{1_{z z}}}\left(u_{x}-x u_{y y}\right)\right)>0
\end{gathered}
$$

We note that if $n_{\text {sun }}$ becomes small and if $u_{x x}>0, u_{y y}<0$, and $\left(v x / I_{1_{z z}}\right)\left(u_{x}-x u_{y y}\right)>0$, then this condition is satisfied, ultimately leading to one hyperbolic stable and unstable mode and one pure oscillatory mode. In all physical setups of interest for asteroid deflection, $n_{\text {sun }}$ is extremely small, even after the normalization, so that this outcome remains. Thus, the nature of the eigenvalues $\pm \sigma_{x}$ and $\pm \sigma_{y}$ do not change between the two equilibria.

For the four-state subspace and the dynamics on it, the characteristic exponents for modes are given by

$$
\begin{aligned}
\sigma_{z}^{2}, & \sigma_{\omega}^{2}=-\frac{1}{2}\left[-\frac{n_{\text {sun }}^{2}\left(I_{1_{y y}}-I_{1_{z z}}\right)\left(I_{1_{z z}}-I_{1_{x x}}\right)}{I_{1_{x x}} I_{1_{y y}}}-u_{z z}\right. \\
& \left.+\frac{v x}{I_{1_{y y}}}\left(u_{x}-x u_{z z}\right)\right] \pm \frac{1}{2}\left\{\left[-\frac{n_{\text {sun }}^{2}\left(I_{1_{y y}}-I_{1_{z z}}\right)\left(I_{1_{z z}}-I_{1_{x x}}\right)}{I_{1_{x x}} I_{1_{y y}}}\right.\right. \\
& \left.-u_{z z}+\frac{v x}{I_{1_{y y}}}\left(u_{x}-x u_{z z}\right)\right]^{2} \\
& -4\left[\frac{n_{\text {sun }}^{2}\left(I_{1_{y y}}-I_{1_{z z}}\right)\left(I_{1_{z z}}-I_{1_{x x}}\right)}{I_{1_{x x}} I_{1_{y y}}}\left(u_{z z}-\frac{v x}{I_{1_{y y}}}\left(u_{x}-x u_{z z}\right)\right)\right. \\
& \left.\left.-n_{\text {sun }}^{2} \frac{\left(I_{1_{y y}}-I_{1_{z z}}+I_{1_{x x}}\right)\left(I_{1_{y y}}-I_{1_{z z}}\right) v x}{I_{1_{y y}}^{2} I_{1_{x x}}}\left(u_{x}-x u_{z z}\right)\right]\right\}^{\frac{1}{2}}
\end{aligned}
$$

We note again that in the case in which $n_{\text {sun }}=0$, elimination of terms in the preceding recovers the bottom part of Eq. (15), plus $\sigma_{\omega}^{2}=0$. Also, if we have a design for the GT that is axisymmetric about the $\hat{\mathbf{i}}$ axis of the GT-fixed frame, aligned with the local vertical for either equilibrium, then $I_{1_{y y}}=I_{1_{z z}}$. This will be assumed later for both the 
two-point-mass (or dumbbell) and ellipsoid GT representations we use herein for the pendulum GT configuration. With this assumption, the elimination of terms from Eq. (20) again exactly recovers the bottom part of Eq. (15) plus $\sigma_{\omega}^{2}=0$. Just for completeness, if both $n_{\text {sun }} \neq 0$ and $I_{1_{y y}} \neq \overline{I_{1 z z}}$ (in reality, perfect symmetry about the $\hat{\mathbf{i}}$ axis cannot be achieved), we may wish to see if the nature of the eigenvalues changes between the equilibria. To have the squares of the characteristic exponents be real numbers, we would need for the quantity in braces in Eq. (20) to be positive. This condition can be expanded as

$$
\begin{aligned}
& \left(\frac{n_{\text {sun }}^{2}\left(I_{1_{y y}}-I_{1_{z z}}\right)\left(I_{1_{z z}}-I_{1_{x x}}\right)}{I_{1_{x x}} I_{1_{y y}}}\right)^{2}+u_{z z}^{2}+\left(\frac{v x}{I_{1_{y y}}}\left(u_{x}-x u_{z z}\right)\right)^{2} \\
& -2 \frac{n_{\text {sun }}^{2}\left(I_{1_{y y}}-I_{1_{z z}}\right)\left(I_{1_{z z}}-I_{1_{x x}}\right)}{I_{1_{x x}} I_{1_{y y}}} u_{z z} \\
& \quad+2 \frac{n_{\text {sun }}^{2}\left(I_{1_{y y}}-I_{1_{z z}}\right)\left(I_{1_{z z}}-I_{1_{x x}}\right) v x}{I_{1_{x x}} I_{1_{y y}}^{2}}\left(u_{x}-x u_{z z}\right) \\
& -2 u_{z z} \frac{v x}{I_{1_{y y}}}\left(u_{x}-x u_{z z}\right)+4 n_{\text {sun }}^{2} \\
& \quad \times \frac{\left(I_{1_{y y}}-I_{1_{z z}}+I_{1_{x x}}\right)\left(I_{1_{y y}}-I_{1_{z z}}\right) v x}{I_{1_{x x}} I_{1_{y y}}^{2}}\left(u_{x}-x u_{z z}\right)>0
\end{aligned}
$$

simplifying to

$$
\begin{aligned}
& {\left[\frac{n_{\text {sun }}^{2}\left(I_{1_{y y}}-I_{1_{z z}}\right)\left(I_{1_{z z}}-I_{1_{x x}}\right)}{I_{1_{x x}} I_{1_{y y}}}-u_{z z}+\frac{v x}{I_{1_{y y}}}\left(u_{x}-x u_{z z}\right)\right]^{2}} \\
& \quad+4 n_{\text {sun }}^{2} \frac{\left(I_{1_{y y}}-I_{1_{z z}}+I_{1_{x x}}\right)\left(I_{1_{y y}}-I_{1_{z z}}\right) v x}{I_{1_{x x}} I_{1_{y y}}^{2}}\left(u_{x}-x u_{z z}\right)>0
\end{aligned}
$$

Now we see that if $n_{\text {sun }}$ is small enough to make the term it appears in within the preceding left square brackets very much smaller than the rest of what is within those brackets, this is satisfied. And if the quantity $v x / I_{1_{y y}}\left(u_{x}-x u_{z z}\right)>0$ and either $I_{1_{y y}}>I_{1_{z z}}$ or $I_{1_{z z}}>I_{1_{y y}}+I_{1_{x x}}>I_{1_{y y}}$, then regardless of the rate $n_{\text {sun }}$, it is guaranteed satisfied. These are statements of sufficiency and not necessity, because the inequality (21) may be satisfied in other ways too. Suppose it is, and let $n_{\text {sun }}$ be small but increasing in magnitude from zero. We expect that with the second desired relative equilibrium, the dynamics of the $Z$ component of position and velocity perturbation were pure oscillatory. That will not change as $n_{\text {sun }}$ first departs from zero, so let the square of the characteristic exponent $\sigma_{z}$ remain negative. If, as $n_{\text {sun }}$ departs from zero, the square of the characteristic exponent $\sigma_{\omega}$ departs from zero in the same negative direction, then we will simply have an additional oscillatory mode. But if it instead departs in the positive direction, we will now have a very slow hyperbolic stable and unstable behavior. This is unlikely to need to be specially addressed, because of how incredibly slow the unstable mode is likely to be, especially as the spacecraft is made closer and closer to axisymmetric, on top of having a slow equilibrium rotation rate.

In general, it is satisfactory to use the same force-feedback control method for the first equilibrium as used for the second equilibrium, as shown in Eq. (17), without modification. Of course within Eq. (17), the uppermost (position perturbation) portion of the nine-element eigenvectors matching with the stable and unstable eigenvalues of hyperbolic modes are different in this case. The characteristic exponent for the hyperbolic modes is also different in this case and are given by Eq. (18) or Eq. (20).

Use of this control force feedback of Eq. (17) on top of the nominal control force gives stability (in the sense of Lyapunov) for all states. However, it may be desired to apply an additional control moment too, to produce behavior in the otherwise indirectly driven angular velocity perturbation that is not just oscillation with unacceptably long time constants. For the first equilibrium, a feedback of the form

$$
\begin{aligned}
\delta \mathbf{m}_{c} & =\left[\begin{array}{ccc}
-g_{\omega 1} I_{1_{x x}} & n_{\text {sun }}\left(I_{1_{z z}}-I_{1_{y y}}\right) & 0 \\
n_{\text {sun }}\left(I_{1_{x x}}-I_{1_{z z}}\right) & -g_{\omega 2} I_{1_{y y}} & 0 \\
0 & 0 & -g_{\omega 3} I_{1_{z z}}
\end{array}\right] \delta \boldsymbol{\omega}_{1} \\
+ & {\left[\begin{array}{ccc}
0 & 0 & 0 \\
0 & 0 & v\left(u_{x}-x u_{z z}\right) \\
0 & -v\left(u_{x}-x u_{y y}\right) & 0
\end{array}\right] \delta \mathbf{r} }
\end{aligned}
$$

with gains $g_{\omega 1}, g_{\omega 2}, g_{\omega 3}>0$ is sufficient for asymptotic stability of the angular velocity perturbation. Here, the rightmost $\delta \mathbf{r}$ term stops the angular velocity dynamics from being one-way-driven by the position perturbation, so that we have clean exponential decay. We might also want to nullify the coupling from this applied control moment into the upper position and velocity system so as not to undo the previous position and velocity oscillation behavior. That necessitates adding another component to the force feedback of

$$
\delta \mathbf{f}_{c_{\omega}}=\left[\begin{array}{c}
0 \\
g_{\omega 3} x \delta \boldsymbol{\omega}_{1_{z}}+\frac{v x}{I_{1_{z z}}}\left(u_{x}-x u_{y y}\right) \delta \mathbf{r}_{y} \\
\frac{n_{\operatorname{sun}} x}{I_{1_{y y}}}\left(I_{1_{x x}}-I_{1_{z z}}\right) \delta \boldsymbol{\omega}_{1_{x}}-g_{\omega 2} x \delta \omega_{1_{y}}+\frac{v x}{I_{y y}}\left(u_{x}-x u_{z z}\right) \delta \mathbf{r}_{z}
\end{array}\right]
$$

If we wish to allow the coupling from position and velocity into angular velocity, we need only omit the $\delta \mathbf{r}$ terms from Eqs. (22) and (23), and if we wish to allow the coupling in the other direction, we do not include Eq. (23) at all. We find that to introduce damping into the position and velocity oscillations, we will wish to allow all of the coupling to remain in the system, and more than that, we will wish to add a rate-feedback term involving the local vertical component of velocity; that is,

$$
\delta \mathbf{f}_{c_{x \mathrm{dot}}}=g_{x}\left[\begin{array}{lll}
\delta \mathbf{r}_{x}^{\prime} & 0 & 0
\end{array}\right]^{T}, \quad g_{x}>0
$$

Finally, for the second equilibrium, we just use the same equations (22-24) after replacing $n_{\text {sun }}$ with zero.

If we now adopt a two-point-mass representation of the pendulum GT for the purpose of finding the mutual potential and its first and second partial derivatives, then we follow the formulation sketched in Appendix A and substitute Eqs. (A2) for the five scalars $x, u_{x}, u_{x x}$, $u_{y y}$, and $u_{z z}$ in all of the preceding. This makes the eigenvalues and control components depend upon the parameter set $\left(\mu, l, r_{d}, v, I_{1_{x x}}, I_{1_{y y}}, I_{1_{z z}}\right)$ for both equilibria. It will be easier to make statements about the eigenvalues and spectral stability of the perturbation dynamics if we can reduce this large number of free parameters. We can approximate $v \approx 1$, noting that almost all mass in the two-body system is concentrated in the target NEO (for a 100$m$ mean equivalent radius and typical densities, the NEO mass is on the order of 10,000,000 t, compared with just tens of metric tons for the GT). We can also choose the most convenient normalization so that the parameter $l$ is always unity. Then the radius of the NEO is a new free parameter, but because both the true dynamics and the perturbation dynamics are invariant with changes in this radius (due to the assumption of a spherical model for the NEO), we can ignore this. With this normalization, we can also substitute the simplified inertia element expressions of Eqs. (A3) into all of the preceding. Only $0<\mu \leq 0.5$ and $R_{\mathrm{NEO}}+\mu \overline{<} r_{d} \leq 2.5 R_{\mathrm{NEO}}$ remain to explore. Herein, the 0.5 and 2.5 are arbitrarily selected ( $\mu$ could be up to one, but this is of little practical interest).

We can conclude from the signs of the right-hand sides of the resulting expressions for the squares of the characteristic exponents that the system is unstable everywhere, with two distinct pure oscillatory modes and one hyperbolic stable and unstable mode (we are supposing that we have perfect axisymmetry so that there is no departure, by the earlier discussion, of $\sigma_{\omega}^{2}$ from zero). For the second equilibrium, the squared characteristic exponent expressions simplify further, and it is apparent that the spectral stability is identical and, in fact, the two undamped oscillatory modes become identical. The only noticeable difference in moving from the second 


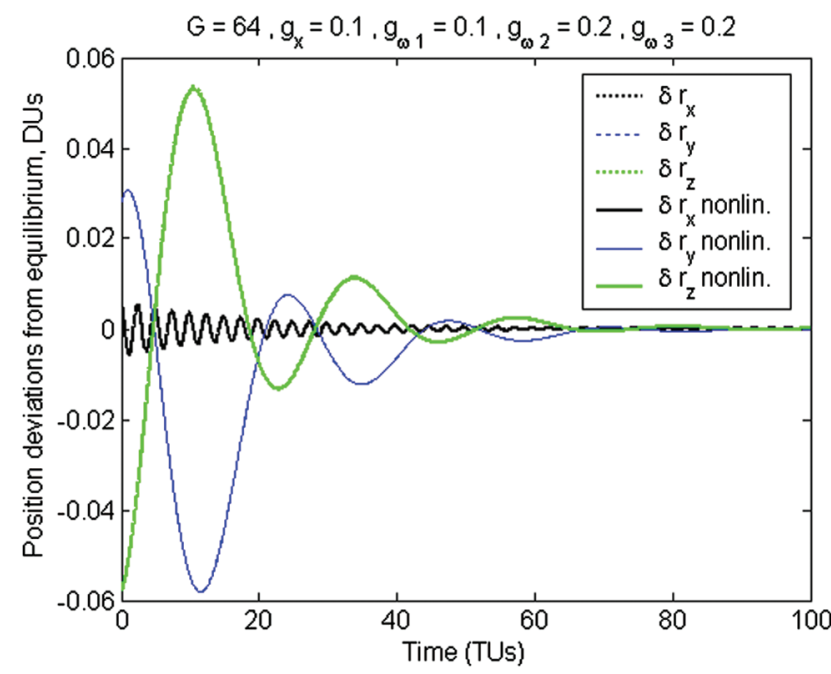

a) Position perturbation
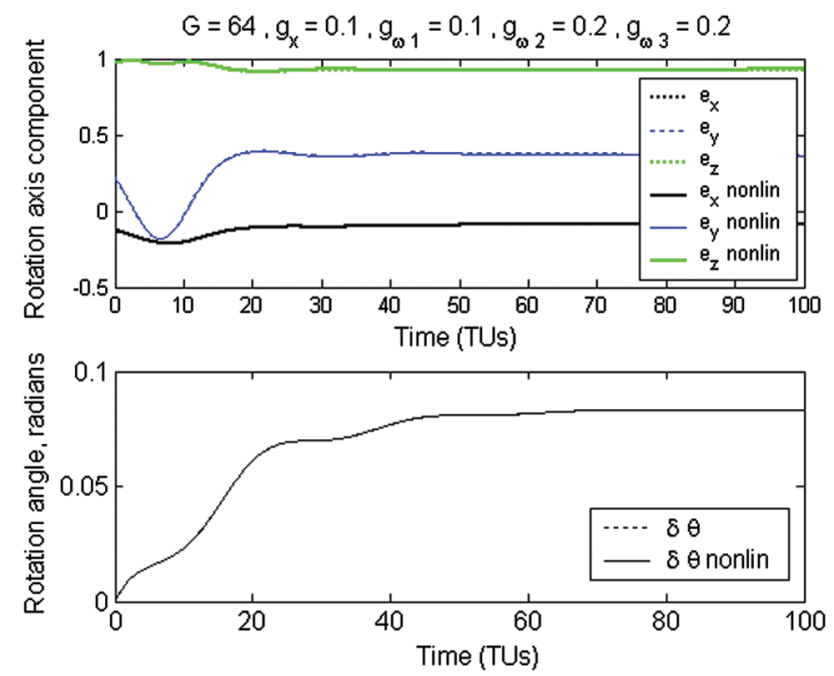

b) Attitude deviation (axis-angle coordinates)

Fig. 3 Comparison of linear and nonlinear normalized system responses for point-mass pendulum GT representation to a randomized initial deviation from the downtrack alignment relative equilibrium.

back to the first equilibrium is that although the eigenvalues $\pm \sigma_{z}$ are not moved, the eigenvalues $\pm \sigma_{x}$ and $\pm \sigma_{y}$ are shifted slightly without changing their type. This is confirmed using MATLAB to do a gridding of the $2 \mathrm{D}$ parameter space within the boundaries $0<\mu \leq$ 0.5 and $R_{\mathrm{NEO}}+\mu<r_{d} \leq 2.5 R_{\mathrm{NEO}}$ to find the eigenvalues at each grid point.

Use of the expressions for the controller resulting from substitution into the nominal control force and the added components of Eqs. (17), (22), and (24) (with no cancellation of coupling) produces the desired closed-loop perturbation-dynamics behavior. For the first equilibrium, Fig. $\underline{3}$ shows the response to initial conditions randomly chosen from within the interval of $[-0.1,0.1]$ for position perturbation, $[-0.01,0.01]$ for velocity perturbation, and $[-0.01,0.01]$ for angular velocity perturbation. Recall that by normalization, the real units have been eliminated and replaced with normalized distance units (DUs) and time units (TUs) and that the normalization length is chosen here as $50 \mathrm{~m}$. For the shown results, we chose to fix the mean motion of the NEO's heliocentric orbit $n_{\text {sun }}$ to that of the Earth (we assumed the 100-m-radius NEO to be on a circular orbit that is well suited to produce an Earth impact, due to similarity to Earth's orbit). The particular point in the parameter space for the shown results is $\mu=0.25$ and $r_{d}=3.44$. The two responses shown are those produced by the linear perturbationdynamics equations with the full $\delta \mathbf{f}_{c}$ and $\delta \mathbf{m}_{c}$ applied and by the original nonlinear equations (5) and (6), with the control force and moment sums $\mathbf{f}_{c_{\mathrm{eq}}}+\delta \mathbf{f}_{c}$ and $\overline{\mathbf{m}}_{c_{\mathrm{eq}}}+\bar{\delta} \mathbf{m}_{c}$ inserted for $\mathbf{f}_{c}$ and $\mathbf{m}_{c}$ on the right-hand side. For the latter results, the equilibrium point in the phase space is subtracted from the raw output, to allow for plotting the two trajectories side by side in the same coordinates of deviation from the equilibrium. We note the presence of an apparent attitude error relative to what the attitude should be to remain consistent with the slow rotation of the towing direction by $n_{\text {sun }}$. In connection to this error, we note that the attitude of the GT with respect to the inertial reference frame is not represented in any of the equations propagated. The attitude behavior will be addressed later.

We can adopt the ellipsoid representation of the pendulum GT instead, as another way of developing the perturbation dynamics in terms of a small number of free parameters, over which stability and behavior can be explored. We follow the formulation sketched for this representation within Appendix A and substitute Eqs. (A10) for the five scalars $x, u_{x}, u_{x x}, u_{y y}$, and $u_{z z}$ and substitute Eqs. ( $\overline{\mathrm{A} 12}$ ) for the inertia elements. Then, again letting $v \approx 1$, we have the eigenvalues of the perturbation dynamics and components of control as a function of the four parameters $\bar{a}, \bar{b}, \bar{c}$, and $r_{d}$, each a normalized distance. If we assume that our GT is axisymmetric (as was the case with the earlier bottom-and-head two-point-mass model), then we have just $\bar{a}, \bar{b}=\bar{c}, r_{d}$. Assuming the most convenient option of normalizing by the largest semi-axis of the ellipsoidal GT so that $\bar{a}$ is always unity, we have just $0<\bar{b} \leq 0.5$ and $R_{\mathrm{NEO}}+1<r_{d} \leq$ $2.5 R_{\mathrm{NEO}}$ to explore.

We observe from the squared characteristic exponent expressions for both equilibria that with $n_{\text {sun }}$ increasing from zero in magnitude relative to the second equilibrium, the values of $\sigma_{x}^{2}$ and $\sigma_{y}^{2}$ change, but their corresponding mode types do not; that is, their corresponding mode types are still hyperbolic and pure oscillatory, respectively. This is due to satisfaction, as confirmed by MATLAB gridding throughout the $2 \mathrm{D}$ parameter space within the preceding given ranges, of the conditions that $\epsilon_{b}$ should not be very much smaller in magnitude than $\epsilon_{a}$ and that $\epsilon_{a}$ should be small enough. The basic nature of the perturbation-dynamics behavior is not only the same between both equilibria, but also consistent between this ellipsoidal GT representation and the earlier point-mass GT representation.

Use of the nominal control force, the controller of Eq. (17), and the damping control moment and control force feedbacks all together again produces the desired perturbation-dynamics behavior. Both the linearized perturbation dynamics and full nonlinear dynamics responses, in both position and attitude, under this control look qualitatively the same as before for the point-mass GT (in Fig. 3). This is seen for responses to initial conditions randomly drawn from the same intervals used before and given the same value of $n_{\text {sun }}$ and other parameters as before. We do note that the nondimensionalization length is, in this case, the largest ellipsoid semi-axis, rather than the boom length, and so is reduced by half relative to the point-mass GT case, which changes the number of nondimensional TUs needed to cover the same actual time duration as before. Qualitatively, the apparent attitude errors are still present in this case.

\section{B. Bar Configuration}

With this configuration, the GT has three parts: the main body containing the power plant, etc., and two thruster nodes, each at the end of a long, lightweight boom, ideally oriented roughly perpendicular to the local vertical. We choose to align the unit vectors $(\hat{\mathbf{i}}, \hat{\mathbf{j}}, \hat{\mathbf{k}})$ for the GT-fixed frame so that $\hat{\mathbf{j}}$ points from the GT centroid along the left boom through the $+Y$ node and so that at either equilibrium, $\hat{\mathbf{i}}$ and $\hat{\mathbf{k}}$ are parallel and perpendicular, respectively, to the towing-direction vector. Then, in the basis of these unit vectors, at either equilibrium, $\mathbf{r}$ and $\partial u / \partial \mathbf{r}$ again have only components $x$ and $u_{x}$ in the $\hat{\mathbf{i}}$ direction, and with the same planar symmetry assumptions for the GT, the matrix $\partial^{2} u / \partial \mathbf{r}^{2}$ again has only diagonal elements $u_{x x}$, $u_{y y}$, and $u_{z z}$.

This means that for both equilibria, all of the top-level formulas for the $A$ and $B$ plant and input matrices, system eigenstructure, etc., presented before for the pendulum configuration still hold. However, 
the inertia elements and values of the five scalars $x, u_{x}, u_{x x}, u_{y y}$, and $u_{z z}$ that we put into all of these equations are different. Because of this, although the conditions listed just after Eq. (19) are still satisfied, still making (19) true so that we get both $\sigma_{x}^{2}$ and $\sigma_{y}^{2}$ to be real numbers, they may no longer have opposing signs and hence may no longer represent modes of different types. Also, although having $n_{\text {sun }}=0$ still recovers the rightmost part of Eq. (15) plus $\sigma_{\omega}^{2}=0$ from Eq. (20), we now certainly do not have axisymmetry about the $\hat{\mathbf{i}}$ axis to give us the same and may have axisymmetry about the $\hat{\mathbf{j}}$ axis instead, with different effects.

If we adopt a three-point-mass model for the bar configuration of the GT, then we follow the formulation outlined in Appendix B and substitute Eqs. (B2) for $x, u_{x}, u_{x x}, u_{y y}$, and $u_{z z}$ in all of the top-level formulas, also using Eqs. (B3) for the inertia elements. Now all of the dynamics can be expressed as functions of the two parameters $\mu$ and $r_{d}$, which we can explore over the chosen ranges of $0<\mu \leq 0.5$ and $R_{\mathrm{NEO}}<r_{d} \leq 2.5 R_{\mathrm{NEO}}$. Note that the lower bound on the latter is closer to the asteroid surface than for the pendulum configuration, possibly allowing for a significantly greater effective towing force on the NEO with this bar configuration.

Examination of the squared characteristic exponent expressions reveals that even though the axisymmetry has changed relative to the pendulum, one still has for the bar that there is no difference between the two equilibria in the modes from the four-state subspace. These are an undamped oscillatory mode and the mode with $\sigma_{\omega}^{2}=0$. As for the five-state subspace, for all parameter values, the signs work out such that we now find this subspace has two unique hyperbolic stable and unstable modes, each having characteristic exponents shifted slightly between the two equilibria; gridding of the parameter space confirms this. This is a fundamental change from the pendulum before, with the new second unstable mode acting in a direction (according to the corresponding eigenvectors) perpendicular to the towing direction. This must necessarily be a direction with some projection onto the equatorial plane of the NEO, and for later rotating NEO models with equatorial ellipticity, this becomes significant from an excitation standpoint.

For the current NEO model and point-mass bar GT, we first nullify the hyperbolic modes by implementing the control force of Eq. (17) again. Two new problems are then presented by adding on top of this the rate-dependent moment and force feedbacks with no cancellation of coupling, after the fashion of Eqs. (22) and (24) for the pendulum GT. First, with the current bar configuration's effective axisymmetry about the $\hat{\mathbf{j}}$ axis, we now have that the $(8,3)$ and $(8,7)$ elements of the linear perturbation-dynamics plant matrix $A$ are effectively zero. Whereas before, the $g_{\omega 2}$ piece of the overall control brought damping through coupling via these elements to the pure oscillatory $Z$ position-velocity mode, now that they are zero, no such damping is introduced. We may choose to introduce it with another direct feedback of the $Z$ component of velocity, just like that of the $X$ component of velocity in Eq. (24). Second, the $(9,2)$ element of the matrix $A$ switches from negative for the pendulum to positive for the bar. This changes the sign of the damping brought through coupling by the $g_{\omega 3}$ piece of the control to the $Y$ position-velocity mode. So now, even after the law of Eq. (17) forces that mode from hyperbolic to pure oscillatory, its amplitude grows unbounded without bringing back cancellation of this coupling of the $g_{\omega 3}$ control into the upper part of the system and, in fact, drives the coupling back the other way. Therefore, one should use the following with Eq. (17):

$$
\begin{gathered}
\delta \mathbf{m}_{c}=\left[\begin{array}{c}
-\frac{g_{\omega 1} \mu}{4} \delta \boldsymbol{\omega}_{1_{x}}+n_{\text {sun }}\left(\frac{\mu}{4}-I_{1_{y y}}\right) \delta \boldsymbol{\omega}_{1_{y}} \\
-g_{\omega 2} I_{1_{y y}} \delta \boldsymbol{\omega}_{1_{y}} \\
-\frac{g_{\omega 3} \mu}{4} \delta \boldsymbol{\omega}_{1_{z}}
\end{array}\right] \\
\delta \mathbf{f}_{c_{x \mathrm{dot}}}=\left[\begin{array}{c}
g_{x} \delta \mathbf{r}_{x}^{\prime} \\
-k g_{\omega 3} r_{d} \delta \boldsymbol{\omega}_{1_{z}} \\
g_{z} \delta \mathbf{r}_{z}^{\prime}
\end{array}\right]
\end{gathered}
$$

with gain $k>0$. This achieves the desired perturbation-dynamics responses. Those responses to the same initial conditions and parameters used in the previous simulations are shown in Fig. 4. The mean motion of the Earth is again used for $n_{\text {sun }}$, and the point in the parameter space is the same as that for the earlier simulation of the two-point-mass pendulum GT configuration and model (i.e., $\mu=$ 0.25 and $\left.r_{d}=3.44\right)$. Both linear perturbation system output and full nonlinear system output, with the equilibrium point subtracted, are shown together, and good agreement between the two is again observed. Apparent steady-state attitude error is also observed.

Now switching to an ellipsoid model for the bar configuration of the GT, Appendix B also gives the expressions for the set of numbers $x, u_{x}, u_{x x}, u_{y y}$, and $u_{z z}$ [Eq. (B4)] and for the inertia elements [Eq. (B5)] for this model and its alignment. Making our substitutions with these and again letting $v \approx 1$, we now have the perturbation dynamics as a function of only the two normalized length parameters $\bar{b}$, and $r_{d}$, with the ranges $0<\bar{b} \leq 0.5$ and $R_{\mathrm{NEO}}+\bar{b}<r_{d} \leq$ $2.5 R_{\mathrm{NEO}}$ to explore (note the new lower bound for collision avoidance).

With nonzero $n_{\text {sun }}$ growing away from zero, the values of the characteristic exponents for the first two modes (in the five-state subspace) only change slightly (and remain of the same type), whereas those for the other two modes (in the four-state subspace) do not change at all. Noting that everywhere in the parameter space outlined previously, $\epsilon_{\bar{b}}$ is larger in magnitude than $\epsilon_{a}$ and both are
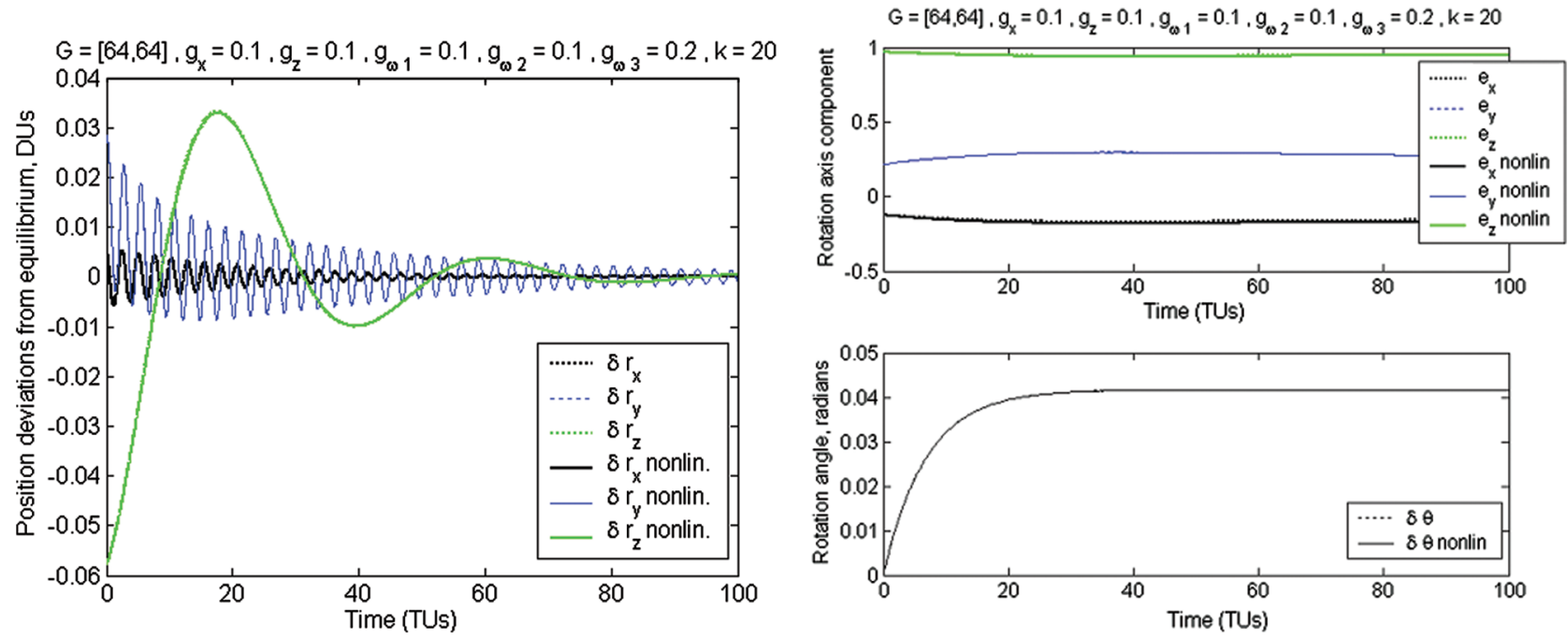

a) Position perturbation

b) Attitude deviation (axis-angle coordinates)

Fig. 4 Comparison of linear and nonlinear normalized system responses for point-mass bar GT representation to a randomized initial deviation from the downtrack alignment relative equilibrium. 
positive, the system is unstable everywhere, with all three nonzero modes distinct from one another: the first two are hyperbolic stable and unstable and the third is undamped oscillatory. The qualitative nature of this modal behavior is therefore unchanged between equilibria and unchanged from the three-point-mass GT representation, even though the parameterization is different. Now the damping control moment and control force that we seek to use changes only slightly to

$$
\begin{gathered}
\delta \mathbf{m}_{c}=\left[\begin{array}{c}
-g_{\omega 1} \frac{1}{5}\left(1+\bar{b}^{2}\right) \delta \boldsymbol{\omega}_{1_{x}}+n_{\text {sun }} \frac{1}{5}\left(1-\bar{b}^{2}\right) \delta \boldsymbol{\omega}_{1_{y}} \\
-g_{\omega 2} \frac{2}{5} \bar{b}^{2} \delta \boldsymbol{\omega}_{1_{y}} \\
-g_{\omega 3} \frac{1}{5}\left(1+\bar{b}^{2}\right) \delta \boldsymbol{\omega}_{1_{z}}
\end{array}\right] \\
\delta \mathbf{f}_{c_{x \mathrm{dot}}}=\left[\begin{array}{c}
g_{x} \delta \mathbf{r}_{x}^{\prime} \\
-k g_{\omega 3} r_{d} \delta \boldsymbol{\omega}_{1_{z}} \\
g_{z} \delta \mathbf{r}_{z}^{\prime}
\end{array}\right]
\end{gathered}
$$

Use of this plus the nominal control force and first control force feedback for mode cancellation [Eq. (17)], all adapted to the eigenvalue expressions of this case, gives the wanted time responses in the states represented within the equations. This was verified for the same mean motion of the Earth and the same initial conditions used in the prior simulations and for the same point in the parameter space used in the earlier simulation of the ellipsoid pendulum GT configuration and model. Concurrent plotting of the linearized perturbation dynamics and full nonlinear dynamics responses shows good agreement between the two and behavior qualitatively similar to that seen in Fig. 4 for the point-mass bar GT. Similar attitude error is present when the other states actually in the equations are all properly controlled.

\section{Homogenous Triaxial Ellipsoid or Oblate Spheroid Representation of NEO}

Both more complex and far more realistic than representing the $\mathrm{NEO}$ as a perfect sphere, for which the rotation state is irrelevant to the dynamics, is representing the NEO as a homogenous-in-density flattened sphere (oblate spheroid) or triaxial ellipsoid. The former is just a special case of the latter, and so we jump directly to dealing with the triaxial ellipsoid hereafter. With the absence of any GT interaction or external forces/torques, the ellipsoid NEO is assumed to be in the energetically stable rotation state that the vast majority of asteroids in nature have reached over time, due to internal energydissipation mechanisms. This is uniform spin with a constant spin rate about the largest moment of inertia principal axis, or the shortest semi-axis, hereafter "spin axis."

Let the attitude rotation matrix $P$ map from the GT-fixed frame to an inertial reference frame and the attitude rotation matrix $T$ map from the NEO-fixed frame to the GT-fixed frame. The rotations represented by these rotation matrices can be fully parameterized by just the angles $\phi, \theta, \psi$, and $\zeta$, shown in Fig. 5, which illustrates the basic setup. These angles and, returning to using units, the relative position vector $\mathbf{R}$ are configuration variables, but we also have momenta variables. These are (all expressed in the GT-fixed frame) relative linear momentum $\mathbf{P}$ and angular momenta $\Gamma_{1}=\mathrm{I}_{1} \Omega_{1}$ and $\Gamma_{2}=T_{2} \Omega_{2}$. The full Hamiltonian for this system may be written in terms of these configuration and momenta variables as follows:

$$
H=\frac{1}{2 m} \mathbf{P}^{T} \mathbf{P}+\frac{1}{2} \Gamma_{1}^{T} \mathrm{I}_{1}^{-1} \Gamma_{1}+\frac{1}{2} \Gamma_{2}^{T} T \mathrm{I}_{2}^{-1} T^{T} \Gamma_{2}+\{-U(\mathbf{R}, \mathrm{T})\}
$$

We can obtain equations of motion for this full two-rigid-body problem, expressed in the GT-fixed frame, that are already reduced using relative coordinates. These equations match with those presented in $[9,10]$ (accounting for the fact that $U$ in this paper is gravitational force potential, not gravitational potential energy).

$$
\begin{array}{cc}
\dot{\mathbf{R}}=\tilde{\mathbf{R}} \boldsymbol{\Omega}_{1}+\frac{\mathbf{P}}{m}, & \dot{\mathbf{P}}=\tilde{\mathbf{P}} \boldsymbol{\Omega}_{1}+\frac{\partial U}{\partial \mathbf{R}} \\
\dot{\Gamma}_{1}=\tilde{\Gamma}_{1} \boldsymbol{\Omega}_{1}+\mathbf{M}_{1}, & \dot{\Gamma}_{2}=\tilde{\Gamma}_{2} \boldsymbol{\Omega}_{1}+\mathbf{M}_{2}
\end{array}
$$

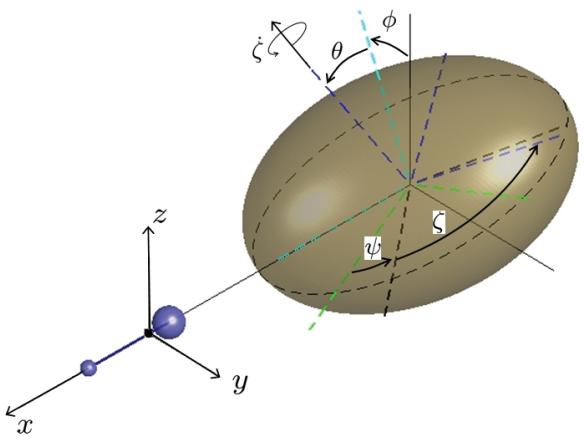

Fig. 5 Illustration of physical configuration of systems with nonspherical NEO representations, including coordinates and relative attitude angles.

$$
\dot{T}=T \tilde{\mathbf{\Omega}}_{2}-\tilde{\mathbf{\Omega}}_{1} T, \quad \dot{P}=P \tilde{\mathbf{\Omega}}_{1}
$$

Note that the force $\mathbf{F}_{1}$ and moment $\mathbf{M}_{1}$ acting on the GT due to mutual gravity, and similar moment $\mathbf{M}_{2}$ acting on the NEO due to mutual gravity, are now determined from a mutual potential that is dependent on relative attitude in addition to relative position. Hence,

$$
\begin{gathered}
\mathbf{F}_{1}=-\frac{\partial U}{\partial \mathbf{R}}, \quad \mathbf{M}_{1}=-\tilde{\mathbf{R}} \frac{\partial U}{\partial \mathbf{R}}-\mathbf{M}_{2} \\
\mathbf{M}_{2}=\tilde{\alpha}_{T} \frac{\partial U}{\partial \alpha_{T}}+\tilde{\beta}_{T} \frac{\partial U}{\partial \beta_{T}}+\tilde{\gamma}_{T} \frac{\partial U}{\partial \gamma_{T}}
\end{gathered}
$$

wherein $\alpha_{T}, \beta_{T}$, and $\gamma_{T}$ are the columns of the rotation matrix $T$. Note that Eqs. (28) can be combined into second-order form as

$$
\ddot{\mathbf{R}}+\tilde{\mathbf{\Omega}}_{1} \mathbf{R}+2 \tilde{\mathbf{\Omega}}_{1} \dot{\mathbf{R}}+\tilde{\boldsymbol{\Omega}}_{1} \tilde{\boldsymbol{\Omega}}_{1} \mathbf{R}=\frac{1}{m} \frac{\partial U}{\partial \mathbf{R}}=-\frac{\mathbf{F}_{1}}{m}
$$

and this plus the left half of Eqs. (29) may be recognized as the equations we started from for the spherical NEO development, with no nonconservative (control) forces present yet. Now normalizing to eliminate units on the Hamiltonian, we find the nondimensional version to be

$$
\mathcal{H}=\frac{1}{2} \mathbf{p}^{T} \mathbf{p}+\frac{1}{2 \nu} \Gamma_{1}^{T} I_{1}^{-1} \Gamma_{1}+\frac{1}{2(1-v)} \Gamma_{2}^{T} T I_{2}^{-1} T^{T} \Gamma_{2}+\{-u(\mathbf{r}, T)\}
$$

Likewise, obtain normalized full two-rigid-body-problem reduced equations of

$$
\begin{gathered}
\mathbf{r}^{\prime}=-\tilde{\omega_{1}} \mathbf{r}+\mathbf{p}, \quad \mathbf{p}^{\prime}=-\tilde{\omega_{1}} \mathbf{p}-\mathbf{f}_{1} \\
\Gamma_{1}^{\prime}=-\tilde{\omega}_{1} \Gamma_{1}+v \mathbf{m}_{1}, \quad \Gamma_{2}^{\prime}=-\tilde{\omega}_{1} \Gamma_{2}+(1-v) \mathbf{m}_{2} \\
T^{\prime}=T \tilde{\omega}_{2}-\tilde{\omega}_{1} T, \quad P^{\prime}=P \tilde{\omega}_{1}
\end{gathered}
$$

with gravitational forces and moments given by

$$
\begin{gathered}
\mathbf{f}_{1}=-\frac{\partial u}{\partial \mathbf{r}}, \quad \mathbf{m}_{1}=-\tilde{\mathbf{r}} \frac{\partial u}{\partial \mathbf{r}}-\mathbf{m}_{2} \\
\mathbf{m}_{2}=\tilde{\alpha}_{T} \frac{\partial u}{\partial \alpha_{T}}+\tilde{\beta}_{T} \frac{\partial u}{\partial \beta_{T}}+\tilde{\gamma}_{T} \frac{\partial u}{\partial \gamma_{T}}
\end{gathered}
$$

Again approximate $v=1$, and with this, $\mathbf{m}_{2}$ is "shut off" so that the rotational behavior of the NEO is just free rigid-body rotational dynamics (free precession) and is unaltered from the assumed constant spin about a fixed spin axis, regardless of what the GT does. We can ignore the right one of Eqs. (35) and set our inertial reference frame to be such that its $+Z$ axis is parallel to the spin axis for either configuration (see Fig. 5). The $P$ matrix can then be expressed as 


$$
P=\left[\begin{array}{ccc}
c(\theta) c(\psi) & c(\phi) s(\psi)+s(\phi) s(\theta) c(\psi) & s(\phi) s(\psi)-c(\phi) s(\theta) c(\psi) \\
-c(\theta) s(\psi) & c(\phi) c(\psi)-s(\phi) s(\theta) s(\psi) & s(\phi) c(\psi)+c(\phi) s(\theta) s(\psi) \\
s(\theta) & -s(\phi) c(\theta) & c(\phi) c(\theta)
\end{array}\right]
$$

where $c$ and $s$ are short for cosine and sine, respectively. With the oblate spheroid NEO model, the rotation phase of the NEO about the spin axis does not change the gravity potential at all, so we can ignore the spin about that axis entirely and set both the $\zeta$ and $\zeta^{\prime}$ indicated in Fig. 5 equal to zero. Then we have that $T=P^{T}$, and because $\omega_{2}=\left[\begin{array}{lll}0 & 0 & \zeta^{\prime}\end{array}\right]^{T}$, both Eqs. (36) are identically the same and simplify down to the scalar equations

$$
\begin{gathered}
\phi^{\prime}=-\omega_{1_{x}}-\tan (\theta) s(\phi) \omega_{1_{y}}+\tan (\theta) c(\phi) \omega_{1_{z}} \\
\theta^{\prime}=-c(\phi) \omega_{1_{y}}-s(\phi) \omega_{1_{z}} \\
\psi^{\prime}=s(\phi) / c(\theta) \omega_{1_{y}}-c(\phi) / c(\theta) \omega_{1_{z}}
\end{gathered}
$$

The second subscript denotes the component of the vector to which it is applied. With the more general ellipsoid NEO model, we have instead that the relative attitude matrix $T$ is the transpose of the righthand side of Eq. (38), with all instances of $\psi$ replaced by $\psi+\zeta$, and we also add back in the equation $\zeta^{\prime}=$ constant $\neq 0$. This plus Eqs. (39) and also

$$
\begin{gathered}
\mathbf{r}^{\prime \prime}+\tilde{\omega}_{1}^{\prime} \mathbf{r}+2 \tilde{\omega}_{1} \mathbf{r}^{\prime}+\tilde{\omega}_{1} \tilde{\omega}_{1} \mathbf{r}=-\mathbf{f}_{1}-\mathbf{f}_{c} \\
I_{1} \omega_{1}^{\prime}+\tilde{\omega}_{1} I_{1} \omega_{1}=v \mathbf{m}_{1}+\mathbf{m}_{c}
\end{gathered}
$$

form the final reduced set of equations of motion for the dynamics, with still undetermined control forces and moments added. We now proceed to examine both the pendulum and bar configurations, but for each of these, we use only the corresponding point-mass representation of the GT, so that our expressions for the mutual gravity potential and for its gradients with respect to relative position and attitude exist in closed (nonseries) forms. In each case, the natural normalized mutual force potential is the weighted sum of components $u_{i}$ due to the whole ellipsoid NEO paired with the $i$ th point mass within the GT. Each such component is found using Eq. (A4) in Appendix $\underline{A}$ and its accompanying definitions of $\Delta(p)$ and of the lower integration bound $\lambda$. However, now the $a \geq b \geq c$ in these expressions are normalized semi-axes belonging to the NEO, and $a$ is assumed aligned with the $+X$ axis of the NEO-fixed frame, and the position vector components $x_{i}, y_{i}$, and $z_{i}$ used throughout these expressions are the components of the position vector $\mathbf{r}_{i}$ from the NEO's center of mass to the $i$ th point mass of the GT, coordinated in the spinning NEO-fixed frame.

\section{A. Pendulum Configuration}

For the pendulum GT, in the previous statements, $i \in\{b, h\}$, and the normalized mutual force potential is

$$
u=(1-\mu) u_{b}+\mu u_{h}
$$

Further, we can express the position vectors from the NEO centroid to each node of the pendulum GT in the NEO-fixed frame as

$$
\begin{gathered}
{\left[\begin{array}{lll}
x_{b} & y_{b} & z_{b}
\end{array}\right]^{T}=\mathbf{r}_{b}=T^{T}(-\mathbf{r}-\mu \hat{\mathbf{i}})} \\
{\left[\begin{array}{lll}
x_{h} & y_{h} & z_{h}
\end{array}\right]^{T}=\mathbf{r}_{h}=T^{T}[-\mathbf{r}+(1-\mu) \hat{\mathbf{i}}]}
\end{gathered}
$$

Then the gradient of interest with respect to relative position is

$$
\begin{aligned}
\frac{\partial u}{\partial \mathbf{r}} & =(1-\mu) \frac{\partial u_{b}}{\partial \mathbf{r}_{b}} \cdot \frac{\partial \mathbf{r}_{b}}{\partial \mathbf{r}}+\mu \frac{\partial u_{h}}{\partial \mathbf{r}_{h}} \cdot \frac{\partial \mathbf{r}_{h}}{\partial \mathbf{r}} \\
& =(1-\mu) \frac{\partial}{\partial \mathbf{r}}\left[(-\mathbf{r}-\mu \hat{\mathbf{i}})^{T} T\right]\left[\begin{array}{l}
-\epsilon_{a_{b}} x_{b} \\
-\epsilon_{b_{b}} y_{b} \\
-\epsilon_{c_{b}} z_{b}
\end{array}\right] \\
& +\mu \frac{\partial}{\partial \mathbf{r}}\left[(-\mathbf{r}+(1-\mu) \hat{\mathbf{i}})^{T} T\right]\left[\begin{array}{l}
-\epsilon_{a_{h}} x_{h} \\
-\epsilon_{b_{h}} y_{h} \\
-\epsilon_{c_{h}} z_{h}
\end{array}\right] \\
& =-(1-\mu) T\left[\begin{array}{ccc}
0 & \epsilon_{b_{b}} & 0 \\
0 & 0 & \epsilon_{c_{c}}
\end{array}\right] T^{T}(\mathbf{r}+\mu \hat{\mathbf{i}}) \\
& -\mu T\left[\begin{array}{ccc}
\epsilon_{a_{h}} & 0 & 0 \\
0 & \epsilon_{b_{h}} & 0 \\
0 & 0 & \epsilon_{c_{h}}
\end{array}\right] T^{T}(\mathbf{r}-(1-\mu) \hat{\mathbf{i}})
\end{aligned}
$$

By a similar approach, the gradients of interest with respect to relative attitude are

$$
\begin{gathered}
\frac{\partial u}{\partial \alpha_{T}}=-(1-\mu)[\mathbf{r}+\mu \hat{\mathbf{i}}] \epsilon_{a_{b}} \alpha_{T}^{T}[\mathbf{r}+\mu \hat{\mathbf{i}}] \\
-\mu[\mathbf{r}-(1-\mu) \hat{\mathbf{i}}] \epsilon_{a_{h}} \alpha_{T}^{T}[\mathbf{r}-(1-\mu) \hat{\mathbf{i}}] \\
\frac{\partial u}{\partial \beta_{T}}=-(1-\mu)[\mathbf{r}+\mu \hat{\mathbf{i}}] \epsilon_{b_{b}} \beta_{T}^{T}[\mathbf{r}+\mu \hat{\mathbf{i}}] \\
-\mu[\mathbf{r}-(1-\mu) \hat{\mathbf{i}}] \epsilon_{b_{h}} \beta_{T}^{T}[\mathbf{r}-(1-\mu) \hat{\mathbf{i}}] \\
\frac{\partial u}{\partial \gamma_{T}}=-(1-\mu)[\mathbf{r}+\mu \hat{\mathbf{i}}] \epsilon_{c_{b}} \gamma_{T}^{T}[\mathbf{r}+\mu \hat{\mathbf{i}}] \\
\quad-\mu[\mathbf{r}-(1-\mu) \hat{\mathbf{i}}] \epsilon_{c_{h}} \gamma_{T}^{T}[\mathbf{r}-(1-\mu) \hat{\mathbf{i}}]
\end{gathered}
$$

Now we seek to design some additional potential components to be added into the potential energy part of the Hamiltonian, thus producing conservative control forces and torques, formed through gradients in the same preceding fashion, within the equations of motion. Informed by earlier study of the spherical NEO cases, we will likely also want to add nonconservative control forces and torques for energy dissipation. Ideally, we would like to use the modified Hamiltonian itself as a candidate Lyapunov function to verify closed-loop stability of the desired equilibrium. Recall that the normalized Hamiltonian written without the portion for the NEO's rotational dynamics (because $v \approx 1$ ) is

$$
\mathcal{H}=\frac{1}{2} \mathbf{p}^{T} \mathbf{p}+\frac{1}{2} \Gamma_{1}^{T} I_{1}^{-1} \Gamma_{1}-u(\mathbf{r}, T)
$$

There are several different ways of expressing the whole state vector $\mathbf{x}$ for the reduced system represented by Eqs. (39-41) and hence of 
formally writing the desired equilibrium state vector $\mathbf{x}_{\mathrm{eq}}$ for the (always assumed hereafter) downtrack alignment equilibrium. We will favor writing it as

$$
\mathbf{x}_{\mathrm{eq}}=\left[\begin{array}{llllll}
\delta \mathbf{r}^{T} & \mathbf{r}^{T} & \delta \boldsymbol{\omega}_{1}^{T} & \delta \phi & \delta \theta & \delta \psi
\end{array}\right]_{\mathrm{eq}}^{T}=\left[\begin{array}{llllll}
\mathbf{0}^{T} & \mathbf{0}^{T} & \mathbf{0}^{T} & 0 & 0 & 0
\end{array}\right]^{T}
$$

Here, the deviations away from the equilibrium are $\delta \mathbf{r}=\mathbf{r}+r_{d} \hat{\mathbf{i}}$, $\delta \omega_{1}=\omega_{1}-n_{\text {sun }} \hat{\mathbf{k}}, \delta \phi=\phi-\phi_{d}, \delta \theta=\theta-\theta_{d}, \delta \psi=\psi-\psi_{d}$ and, equivalently, in the inertial frame attached to the NEO's assumed invariant spin axis,

$$
\begin{gathered}
\delta \mathbf{x}_{b}=P(-\mathbf{r}-\mu \hat{\mathbf{i}})-\left(r_{d}-\mu\right) P_{d} \hat{\mathbf{i}} \\
\delta \mathbf{x}_{h}=P[-\mathbf{r}+(1-\mu) \hat{\mathbf{i}}]-\left(r_{d}+1-\mu\right) P_{d} \hat{\mathbf{i}}
\end{gathered}
$$

where $P_{d}$ is given by Eq. (하) with the desired angles $\phi_{d}$, etc., used instead. We seek to make local minima in the total energy by placing "wells" in the potential energy, in which we want each piece of the dumbbell GT to remain. This is equivalent to increasing the potential energy everywhere by some piecewise smooth function of the appropriate deviations away from equilibrium, having zero value and zero slope at the origin. Note that if we use the deviations of $\delta \mathbf{x}_{b}$ and $\delta \mathbf{x}_{h}$, which are position- and attitude-dependent only, we ensure that the revised potential energy is not dependent on velocity variables (as it should not be). We choose a function of the form

$$
\left.\begin{array}{ccc}
k_{2}-\frac{k_{1}}{\left(\| \delta \mathbf{x}_{i}-x_{\mathrm{DZ}}\right)^{3} \|} & : & \left\|\delta \mathbf{x}_{i}\right\|-x_{\mathrm{DZ}}>\left(3 \varepsilon k_{1}\right)^{\frac{1}{5}} \\
\frac{\left(\left\|\delta \mathbf{x}_{i}\right\|-x_{\mathrm{DZ}}\right)^{2}}{2 \varepsilon} & : & 0<\left\|\delta \mathbf{x}_{i}\right\|-x_{\mathrm{DZ}} \leq\left(3 \varepsilon k_{1}\right)^{\frac{1}{5}} \\
0 & : & \left\|\delta \mathbf{x}_{i}\right\|-x_{\mathrm{DZ}} \leq 0
\end{array}\right\}
$$

with $i \in\{b, h\}$, which is illustrated in Fig. 6. The shape of the well is controlled by the three free parameters $x_{\mathrm{DZ}}, \varepsilon$, and $k_{1}$, with the latter two together defining the depth $k_{2}$. To create a clean datum below which this function depresses the potential energy, we might also temporarily (we will undo this in a moment) completely cancel the natural mutual gravity potential by subtracting a copy of it, denoted by an overbar. Then we have the new modified mutual force potential as

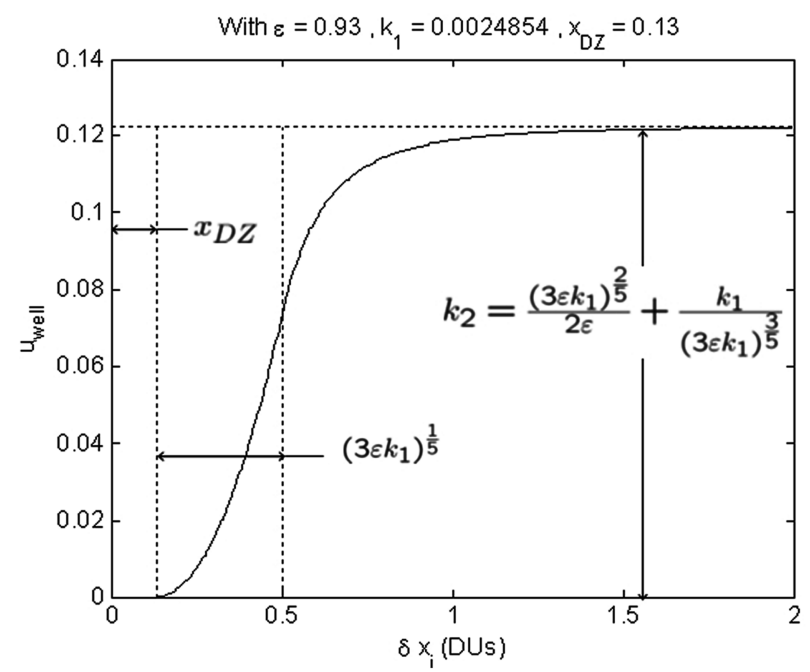

Fig. 6 Cross section of the artificial potential well function.

$$
\begin{gathered}
u_{\text {mod }}=(1-\mu)\left(u_{b}-\bar{u}_{b}\right)+\left\{\begin{array}{c}
-k_{2}+\frac{k_{1}}{\left(\left\|\delta \mathbf{x}_{b}\right\|-x_{\mathrm{DZ}}\right)^{3}} \\
-\frac{\left(\left\|\delta \mathbf{x}_{b}\right\|-x_{\mathrm{DZ}}\right)^{2}}{2 \varepsilon} \\
0
\end{array}\right\} \\
+\mu\left(u_{h}-\bar{u}_{h}\right)+\left\{\begin{array}{c}
-k_{2}+\frac{k_{1}}{\left(\left\|\delta \mathbf{x}_{h}\right\|-x_{\mathrm{DZ}}\right)^{3}} \\
-\frac{\left(\left\|\delta \mathbf{x}_{h}\right\|-x_{\mathrm{DZ}}\right)^{2}}{2 \varepsilon} \\
0
\end{array}\right\}
\end{gathered}
$$

From here on we omit writing the conditions for the selection of which piece of the piecewise function to use, which should be understood to still be in effect. Rather than replace the previously present natural mutual force potential of Eq. (42) with the modified mutual force potential of Eq. (53) and processing gradients as before, it is simpler to find the gradients of the new parts of Eq. (53) with respect to the position deviations in the inertial frame, transform the resulting forces back into the GT-fixed frame, and apply them directly to the applicable end of the GT. We can write the closed-loop set of reduced equations of motion in easily computable first-order form:

$$
\begin{gathered}
\delta \mathbf{r}^{\prime}=\mathbf{r}^{\prime} \\
\mathbf{r}^{\prime \prime}-\left[I_{1}^{-1}\left\{-\left(\delta \boldsymbol{\omega}_{1}+n_{\mathrm{sun}} \hat{\mathbf{k}}\right) \times I_{1}\left(\delta \boldsymbol{\omega}_{1}+n_{\mathrm{sun}} \hat{\mathbf{k}}\right)+v \mathbf{m}_{1}+\mathbf{m}_{c}\right\}\right] \\
\times\left(\delta \mathbf{r}-r_{d} \hat{\mathbf{i}}\right)-2\left(\delta \boldsymbol{\omega}_{1}+n_{\mathrm{sun}} \hat{\mathbf{k}}\right) \times \mathbf{r}^{\prime}-\left(\delta \boldsymbol{\omega}_{1}+n_{\mathrm{sun}} \hat{\mathbf{k}}\right) \\
\times\left(\delta \boldsymbol{\omega}_{1}+n_{\mathrm{sun}} \hat{\mathbf{k}}\right) \times\left(\delta \mathbf{r}-r_{d} \hat{\mathbf{i}}\right)-\mathbf{f}_{1}-\mathbf{f}_{c} \\
\delta \boldsymbol{\omega}_{1}^{\prime}=I_{1}^{-1}\left\{-\left(\delta \boldsymbol{\omega}_{1}+n_{\mathrm{sun}} \hat{\mathbf{k}}\right) \times I_{1}\left(\delta \boldsymbol{\omega}_{1}+n_{\mathrm{sun}} \hat{\mathbf{k}}\right)+v \mathbf{m}_{1}+\mathbf{m}_{c}\right\} \\
\delta \phi^{\prime}=-\delta \boldsymbol{\omega}_{1_{x}}-\tan \left(\delta \theta+\theta_{d}\right)\left[s\left(\delta \phi+\phi_{d}\right) \delta \boldsymbol{\omega}_{1_{y}}\right. \\
\left.-c\left(\delta \phi+\phi_{d}\right)\left(\delta \boldsymbol{\omega}_{1_{z}}+n_{\mathrm{sun}}\right)\right]-s\left(\theta_{d}\right) c\left(\phi_{d}\right) n_{\mathrm{sun}} / c\left(\theta_{d}\right) \\
\delta \theta^{\prime}=-c\left(\delta \phi+\phi_{d}\right) \delta \boldsymbol{\omega}_{1_{y}}-s\left(\delta \phi+\phi_{d}\right)\left(\delta \boldsymbol{\omega}_{1_{z}}+n_{\mathrm{sun}}\right)+s\left(\phi_{d}\right) n_{\mathrm{sun}} \\
\delta \psi^{\prime}=s\left(\delta \phi+\phi_{d}\right) / c\left(\delta \theta+\theta_{d}\right) \delta \boldsymbol{\omega}_{1_{y}} \\
-c\left(\delta \phi+\phi_{d}\right) / c\left(\delta \theta+\theta_{d}\right)\left(\delta \boldsymbol{\omega}_{1_{z}}+n_{\mathrm{sun}}\right)+c\left(\phi_{d}\right) n_{\mathrm{sun}} / c\left(\theta_{d}\right)
\end{gathered}
$$

For the natural forces and moments in this, one substitutes Eqs. (4548) into Eqs. (37) and then makes the replacements $\mathbf{r} \rightarrow \delta \mathbf{r}-\overrightarrow{r_{d}} \hat{\mathbf{i}}$, $\bar{\phi} \rightarrow \delta \phi+\phi_{d}, \quad \bar{\theta} \rightarrow \delta \theta+\theta_{d}, \quad$ and $\quad \psi \mapsto \delta \psi+\psi_{d} \quad$ in the result. Incorporating nonconservative rate feedbacks chosen with knowledge of what was needed earlier for the spherical NEO and pendulum GT scenario, one also uses these control forces and moments in the preceding equations:

$$
\begin{gathered}
\mathbf{f}_{c}=\frac{\partial \bar{u}}{\partial \mathbf{r}}+P^{T}\left\{\begin{array}{c}
\frac{-3 k_{1}}{\left(\left\|\delta \mathbf{x}_{b}\right\|-x_{\mathrm{DZ}}\right)^{5}}-\frac{1}{\varepsilon} \\
0
\end{array}\right\}\left(\delta \mathbf{x}_{b}-\frac{x_{\mathrm{DZ}} \delta \mathbf{x}_{b}}{\left\|\delta \mathbf{x}_{b}\right\|}\right) \\
+P^{T}\left\{\begin{array}{c}
\frac{-3 k_{1}}{\left(\left\|\delta \mathbf{x}_{h}\right\|-x_{\mathrm{DZ}}\right)^{5}} \\
-\frac{1}{\varepsilon} \\
0
\end{array}\right\}\left(\delta \mathbf{x}_{h}-\frac{x_{\mathrm{DZ}} \delta \mathbf{x}_{h}}{\left\|\delta \mathbf{x}_{h}\right\|}\right)+g_{\mathrm{vel}} \mathbf{r}^{\prime} \\
g_{\mathrm{vel}}>0
\end{gathered}
$$




$$
\begin{aligned}
& \mathbf{m}_{c}=v\left\{\tilde{\mathbf{r}} \frac{\partial \bar{u}}{\partial \mathbf{r}}+\tilde{\alpha}_{T} \frac{\partial \bar{u}}{\partial \alpha_{T}}+\tilde{\beta}_{T} \frac{\partial \bar{u}}{\partial \beta_{T}}+\tilde{\gamma}_{T} \frac{\partial \bar{u}}{\partial \gamma_{T}}\right\} \\
& -\mu \hat{\mathbf{i}} P^{T}\left\{\begin{array}{c}
\frac{-3 k_{1}}{\left(\left\|\delta \mathbf{x}_{b}\right\|-x_{\mathrm{DZ}}\right)^{5}} \\
-\frac{1}{\varepsilon} \\
0
\end{array}\right\}\left(\delta \mathbf{x}_{b}-\frac{x_{\mathrm{DZ}} \delta \mathbf{x}_{b}}{\left\|\delta \mathbf{x}_{b}\right\|}\right) \\
& +(1-\mu) \hat{\mathbf{i}} P^{T}\left\{\begin{array}{c}
\frac{-3 k_{1}}{\left(\left\|\delta \mathbf{x}_{h}\right\|-x_{\mathrm{DZ}}\right)^{5}} \\
-\frac{1}{\varepsilon} \\
0
\end{array}\right\}\left(\delta \mathbf{x}_{h}-\frac{x_{\mathrm{DZ}} \delta \mathbf{x}_{h}}{\left\|\delta \mathbf{x}_{h}\right\|}\right) \\
& +\left[\begin{array}{c}
-g_{\omega 1} I_{1_{x x}} \delta \omega_{1_{x}}+n_{\mathrm{sun}}\left(I_{1_{z z}}-I_{1_{y y}}\right) \delta \omega_{1_{y}} \\
n_{\mathrm{sun}}\left(I_{1_{x x}}-I_{1_{z z}}\right) \delta \omega_{1_{x}}-g_{\omega 2} I_{1_{y y}} \delta \omega_{1_{y}} \\
-g_{\omega 3} I_{1_{z z}} \delta \omega_{1_{z}}
\end{array}\right]
\end{aligned}
$$

wherein we again substitute Eqs. (45-48) into the indicated gradients of the cancellation artificial potential $\overline{\bar{u}}$, substitute Eqs. (51) into the indicated gradients of the artificial wells, and perform the same replacements on the results. In all of the preceding, we simply eliminate all $\bar{u}$ terms to undo the cancellation of the natural potential. Note that such cancellation is not feasible in practice anyway, because it would require highly accurate modeling of the mass distribution of the NEO, including internal density variations, and taxing online processing of the detailed potential-field calculations. We may also simply set $n_{\text {sun }}=0$ throughout to recover the inertially fixed second equilibrium and set $\zeta=0$ throughout to handle just the oblate spheroid NEO, rather than the more general triaxial ellipsoid NEO case.

For brevity, we do not intend to write out a proof of stability for the full closed-loop system of Eqs. (54-59) under the action of the control of Eqs. (60) and (61). Rather, we include here only a sketch of a proof, which is for stability in the sense of Lyapunov (not asymptotic) and that only locally about the equilibrium $\mathbf{x}_{\mathrm{eq}}=0_{12 \times 1}$. For this, we make the further assumptions of $n_{\text {sun }}=0$ (thereby limiting ourselves to the case of the second desired relative equilibrium between the NEO and GT) and of perfect cancellation of the $u$ with $\bar{u}$ within the control. Although the latter is not achievable in reality, as mentioned previously, the depth of the artificial potential wells and the slope of their function of the magnitude of node displacement from the desired node position can be made large enough through selection of the $\varepsilon$ and $k_{1}$ parameters that the natural potential field of $u$ can be considered a small perturbation to the system, which does not fundamentally alter the result. A similar argument applies for the typically quite small value of $n_{\text {sun }}$ introduced when moving back to the first desired relative equilibrium. The domain

$$
D \subset \mathbb{R}^{9} \times[0,2 \pi) \times\left(-\frac{\pi}{2}, \frac{\pi}{2}\right) \times[0,2 \pi)
$$

of interest, which contains the equilibrium point, is defined by

$$
D=\left\{\mathbf{x}:\left\|\delta \mathbf{x}_{b}\right\|-x_{\mathrm{DZ}} \leq\left(3 \varepsilon k_{1}\right)^{\frac{1}{5}},\left\|\delta \mathbf{x}_{h}\right\|-x_{\mathrm{DZ}} \leq\left(3 \varepsilon k_{1}\right)^{\frac{1}{5}}\right\}
$$

which is restrictive only of the position and attitude deviations through Eqs. (51). Then the continuously differentiable Lyapunov function $V: D \rightarrow \mathbb{R}$, based on modification of the prior normalized Hamiltonian of Eq. (49) consistent with introduction of the potential wells and written in the deviation states, is

$$
\begin{aligned}
V= & \frac{1}{2}\left[\mathbf{r}^{\prime}+\delta \boldsymbol{\omega}_{1} \times\left(\delta \mathbf{r}-r_{d} \hat{\mathbf{i}}\right)\right]^{T}\left[\mathbf{r}^{\prime}+\delta \boldsymbol{\omega}_{1} \times\left(\delta \mathbf{r}-r_{d} \hat{\mathbf{i}}\right)\right] \\
& +\frac{1}{2} \delta \boldsymbol{\omega}_{1}^{T} I_{1} \delta \boldsymbol{\omega}_{1}+\left\{\begin{array}{c}
k_{2}-\frac{k_{1}}{\left(\left\|\delta \mathbf{x}_{b}\right\|-x_{\mathrm{DZ})^{3}}\right.} \\
\frac{\left(\left\|\delta \mathbf{x}_{b}\right\|-x_{\mathrm{DZ}}\right)^{2}}{2 \varepsilon} \\
0
\end{array}\right\}+\left\{\begin{array}{c}
k_{2}-\frac{k_{1}}{\left(\left\|\delta \mathbf{x}_{h}\right\|-x_{\mathrm{DZ})^{3}}\right.} \\
\frac{\left(\left\|\delta \mathbf{x}_{h}\right\|-x_{\mathrm{DZ}}\right)^{2}}{2 \varepsilon} \\
0
\end{array}\right\}
\end{aligned}
$$

This satisfies $V>0$ everywhere in $D$ other than in the compact invariant set formed by points concurrently satisfying $\mathbf{r}^{\prime}=0$, $\delta \boldsymbol{\omega}_{1}=0,\left\|\delta \mathbf{x}_{b}\right\| \leq x_{\mathrm{DZ}}$, and $\left\|\delta \mathbf{x}_{h}\right\| \leq x_{\mathrm{DZ}}$, wherein $V=0$. This set includes the equilibrium point $\mathbf{x}_{\mathrm{eq}}$. Next taking the gradients of this $V$ with respect to the states and taking the dot product of that with Eqs. (54-59) using the control gives, after considerable simplification involving cancellation of terms and the use of double-angle formulas for combining the products of gradients of $V$ with respect to the attitude angles and the rates in those angles, that $\dot{V} \leq 0$ in $D$. Therefore, the equilibrium in the dead zone is rendered locally Lyapunov stable using this control.

The responses shown in Figs. 7 and $\underline{8}$ are to the same initial perturbations for position, linear velocity, and angular velocity used in the prior pendulum point-mass GT simulations. In addition, there are initial perturbations randomly drawn from the interval $[-0.05,0.05]$ radians in each attitude angle, away from the corresponding initial desired angle. The initial values for the desired attitude angles are $\phi_{d}=\pi / 5, \theta_{d}=\pi / 4$, and $\psi_{d}=\pi / 3$, all in radians. Note that these desired angles are themselves time-varying
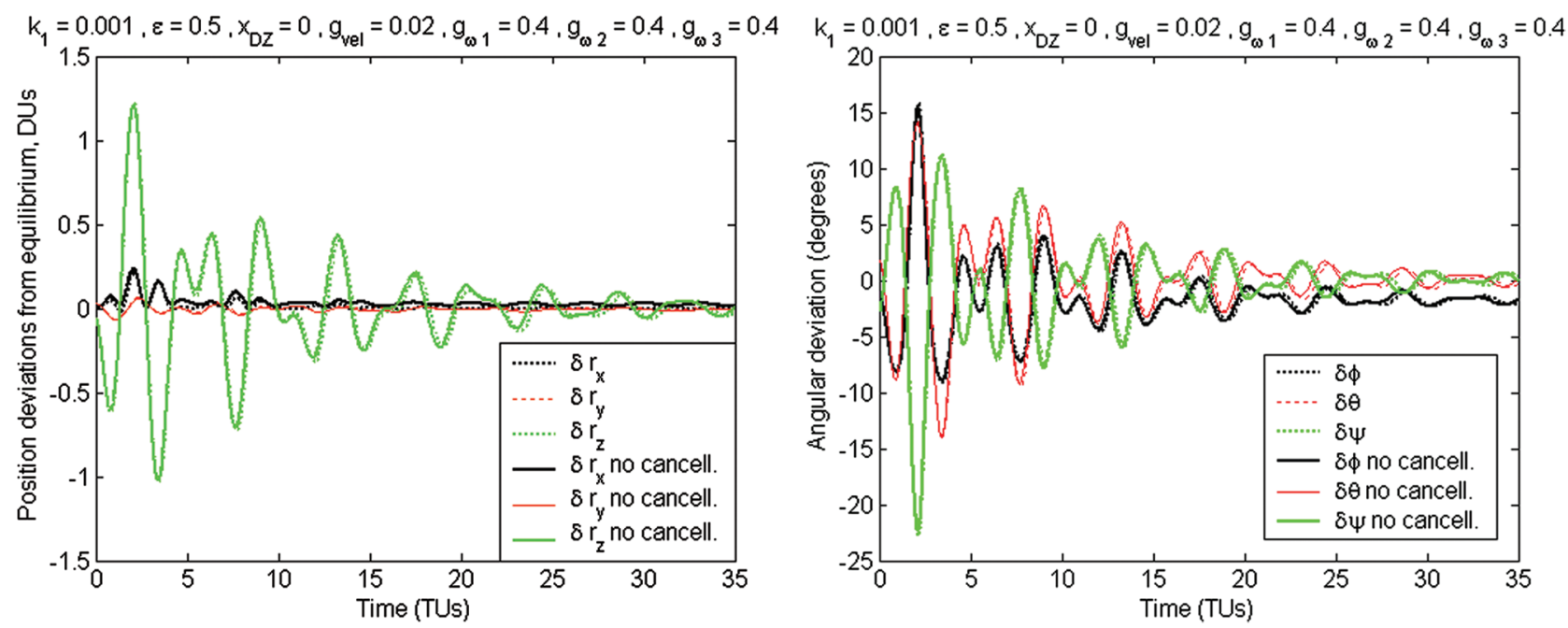

a) Perturbation of position of NEO centroid from that desired for down-track alignment relative equilibrium, coordinated within the GT-fixed frame

b) Deviation of relative attitude angles from desired relative attitude angles for down-track alignment relative equilibrium

Fig. 7 Nonlinear normalized system state responses for two-point-mass pendulum GT representation with triaxial ellipsoid NEO. 

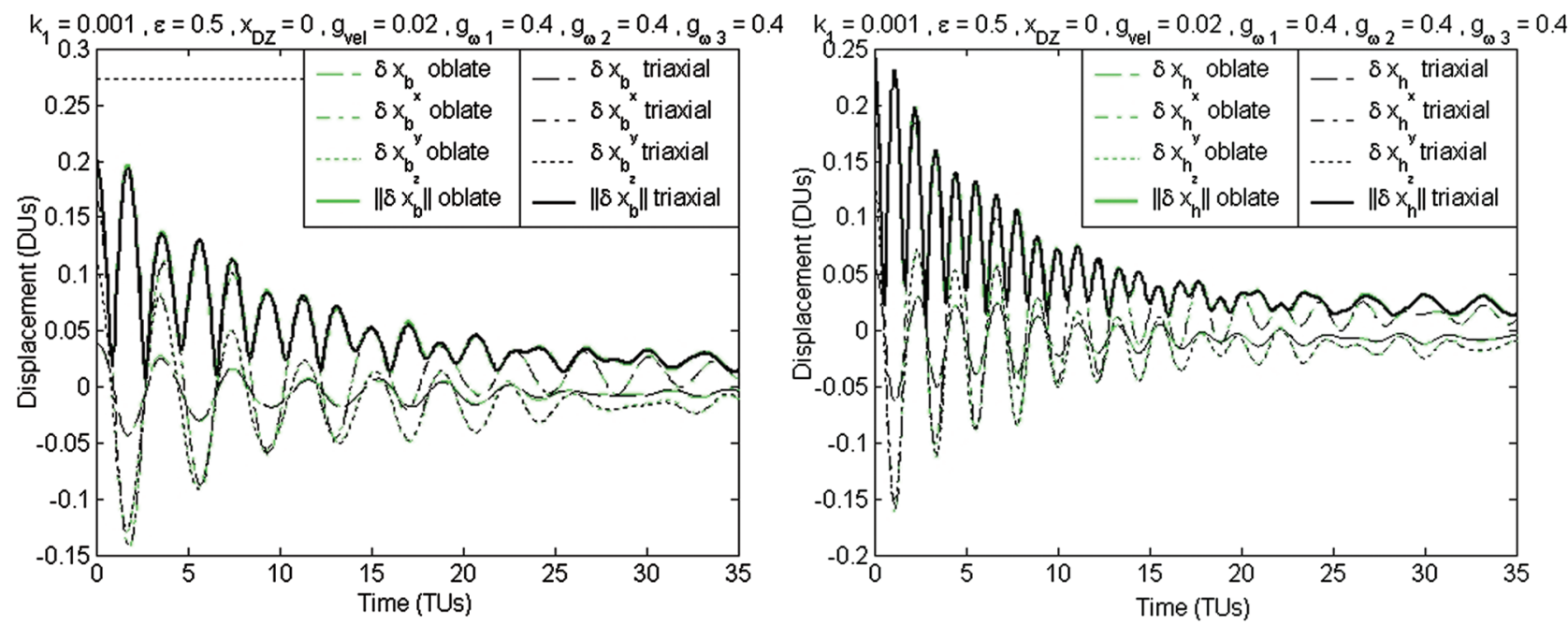

a) Body node

b) Head node

Fig. 8 Nonlinear normalized system response in position error of the GT nodes from their desired position in the inertial frame fixed to the NEO spin axis; with no cancellation of natural potential for the downtrack alignment relative equilibrium and for two-point-mass pendulum GT with both oblate spheroid NEO and triaxial ellipsoid NEO.

in a way matching with any nonzero $n_{\text {sun }}$ value, according to

$$
\begin{gathered}
\phi_{d}^{\prime}=s\left(\theta_{d}\right) c\left(\phi_{d}\right) n_{\mathrm{sun}} / c\left(\theta_{d}\right), \quad \theta_{d}^{\prime}=-s\left(\phi_{d}\right) n_{\mathrm{sun}} \\
\psi_{d}^{\prime}=-c\left(\phi_{d}\right) n_{\mathrm{sun}} / c\left(\theta_{d}\right)
\end{gathered}
$$

These are really additional equations of motion propagated for the results set shown. The same heliocentric mean motion of Earth value for $n_{\text {sun }}$ and the same point in the parameter space for the two-pointmass dumbbell GT used previously are used again here.

In Fig. 8, responses are shown both for an oblate spheroid representation of the NEO with semi-axes of $a=b=112 \mathrm{~m}$ and $c=80 \mathrm{~m}$ and for a triaxial ellipsoid NEO representation with semiaxes of $a=125, b=100$, and $c=80$, all in meters. These dimensions keep the total NEO volume and (with the same assumed density) mass comparable to the NEO volume and mass in all 100-mradius spherical NEO model cases with results shown previously. In addition, the ellipsoid NEO spins with the rate $\zeta^{\prime}$, matching a 2-h rotation period. This is rapid, but not rapid enough to approach the limiting rotation rate for fission of the body [11]. The similar response curves shown reveal that for these cases, all without cancellation of natural potential, the effects of the equatorial ellipticity of the target body persistently exciting the closed-loop dynamics are easily absorbed by the control scheme. In Fig. 7, results are shown only for the more interesting and general ellipsoid NEO, and results are shown side by side, with and without cancelling the natural potential, revealing minimal degradation in performance in moving to the feasible control scheme.

Note that different gains are used here, compared with the previously shown results sets. Any system design constraints are not taken into account yet for gain selection. Here, the values for the gains and the well parameters are chosen merely for illustrative purposes, to reveal qualitative behavior and to give convergence to the desired equilibrium over a span long enough for any effects of the ellipsoid NEO gravity field's rotation to be observed. This translates to near elimination of initial 10.1- and 12.4-m displacements of the body and head, respectively, from their desired places in inertial space, plus mitigation of the other errors, within the span of about $4 \mathrm{~h}$. Note from the concurrent plots (for the ellipsoid NEO case) of both inertial-frame position errors and the actual GT-fixed frame states used that although a zero state vector must produce zero inertialframe position errors, the converse does not hold. That is, near-zero inertial-frame position errors can result from a nonzero state vector, with nonzero attitude-angle deviation about the GT axis of symmetry, in particular. It is the inertial-frame behavior that is really of interest though and that is effectively eliminated here as the system seeks, through the dissipative feedback mechanisms, the lowest energy configuration consistent with our artificially modified potential.

\section{B. Bar Configuration}

For the bar configuration of the GT, in the discussion just before the start of Sec. III.A, we let $i \in\{b,+n,-n\}$, to have three potential contributions from the three point masses representing the GT. The normalized mutual potential is then given by

$$
u=(1-\mu) u_{b}+\frac{\mu}{2} u_{+n}+\frac{\mu}{2} u_{-n}
$$

and in the calculation of each potential contribution $u_{i}$, we use the position vector in the NEO-fixed frame of the matching point mass within the GT, written as

$$
\begin{gathered}
{\left[\begin{array}{lll}
x_{b} & y_{b} & z_{b}
\end{array}\right]^{T}=\mathbf{r}_{b}=-T^{T} \mathbf{r}} \\
{\left[\begin{array}{lll}
x_{+n} & y_{+n} & z_{+n}
\end{array}\right]^{T}=\mathbf{r}_{+n}=T^{T}\left(-\mathbf{r}+\frac{1}{2} \hat{\mathbf{j}}\right)} \\
{\left[\begin{array}{lll}
x_{-n} & y_{-n} & z_{-n}
\end{array}\right]^{T}=\mathbf{r}_{-n}=T^{T}\left(-\mathbf{r}-\frac{1}{2} \hat{\mathbf{j}}\right)}
\end{gathered}
$$

This again leads to, using the chain rule and combination of tensors, the following expressions for the gradients of the normalized mutual potential:

$$
\begin{array}{r}
\frac{\partial u}{\partial \mathbf{r}}=-(1-\mu) T \operatorname{diag}\left\{\left[\begin{array}{l}
\epsilon_{a_{b}} \\
\epsilon_{b_{b}} \\
\epsilon_{c_{b}}
\end{array}\right]\right\} T^{T} \mathbf{r} \\
-\frac{\mu}{2} T \operatorname{diag}\left\{\left[\begin{array}{c}
\epsilon_{a_{+n}} \\
\epsilon_{b_{+n}} \\
\epsilon_{c_{+n}}
\end{array}\right]\right\} T^{T}\left(\mathbf{r}-\frac{1}{2} \hat{\mathbf{j}}\right) \\
-\frac{\mu}{2} T \operatorname{diag}\left\{\left[\begin{array}{c}
\epsilon_{a_{-n}} \\
\epsilon_{b_{-n}} \\
\epsilon_{c_{-n}}
\end{array}\right]\right\} T^{T}\left(\mathbf{r}+\frac{1}{2} \hat{\mathbf{j}}\right)
\end{array}
$$




$$
\begin{aligned}
& \frac{\partial u}{\partial \alpha_{T}}=-(1-\mu) \epsilon_{a_{b}} \alpha_{T}^{T} \mathbf{r}-\frac{\mu}{2}\left[\mathbf{r}-\frac{1}{2} \hat{\mathbf{j}}\right] \epsilon_{a_{+n}} \alpha_{T}^{T}\left[\mathbf{r}-\frac{1}{2} \hat{\mathbf{j}}\right] \\
&- \frac{\mu}{2}\left[\mathbf{r}+\frac{1}{2} \hat{\mathbf{j}}\right] \epsilon_{a_{-n}} \alpha_{T}^{T}\left[\mathbf{r}+\frac{1}{2} \hat{\mathbf{j}}\right] \\
& \frac{\partial u}{\partial \beta_{T}}=-(1-\mu) \mathbf{r} \epsilon_{b_{b}} \beta_{T}^{T} \mathbf{r}-\frac{\mu}{2}\left[\mathbf{r}-\frac{1}{2} \hat{\mathbf{j}}\right] \epsilon_{b_{+n}} \beta_{T}^{T}\left[\mathbf{r}-\frac{1}{2} \hat{\mathbf{j}}\right] \\
&-\frac{\mu}{2}\left[\mathbf{r}+\frac{1}{2} \hat{\mathbf{j}}\right] \epsilon_{b_{-n}} \beta_{T}^{T}\left[\mathbf{r}+\frac{1}{2} \hat{\mathbf{j}}\right] \frac{\partial u}{\partial \gamma_{T}}=-(1-\mu) \mathbf{r} \epsilon_{c_{b}} \gamma_{T}^{T} \mathbf{r}-\frac{\mu}{2}\left[\mathbf{r}-\frac{1}{2} \hat{\mathbf{j}}\right] \epsilon_{c_{+n}} \gamma_{T}^{T}\left[\mathbf{r}-\frac{1}{2} \hat{\mathbf{j}}\right] \\
& \quad+\frac{\mu}{2}\left[\mathbf{r}+\frac{1}{2} \hat{\mathbf{j}}\right] \epsilon_{c_{-n}} \gamma_{T}^{T}\left[\mathbf{r}+\frac{1}{2} \hat{\mathbf{j}}\right]
\end{aligned}
$$

We can follow a similar approach as for the pendulum configuration to introduce two artificial potential wells, this time centered at the locations for the two outboard thruster nodes. We define the position deviations of those nodes from their desired positions, expressed in the inertial frame attached to the NEO's assumed invariant spin axis, as

$$
\begin{aligned}
\delta \mathbf{x}_{+n} & =P\left(-\mathbf{r}+\frac{1}{2} \hat{\mathbf{j}}\right)-P_{d}\left(r_{d} \hat{\mathbf{i}}+\frac{1}{2} \hat{\mathbf{j}}\right) \\
\delta \mathbf{x}_{-n} & =P\left(-\mathbf{r}-\frac{1}{2} \hat{\mathbf{j}}\right)-P_{d}\left(r_{d} \hat{\mathbf{i}}-\frac{1}{2} \hat{\mathbf{j}}\right)
\end{aligned}
$$

Then we use these two deviations as arguments for the same basic function of Eq. (52) and obtain the new modified mutual force potential of

$$
\begin{gathered}
u_{\text {mod }}=(1-\mu)\left(u_{b}-\bar{u}_{b}\right)+\frac{\mu}{2}\left(u_{+n}-\bar{u}_{+n}\right)+\frac{\mu}{2}\left(u_{-n}-\bar{u}_{-n}\right) \\
+\left\{\begin{array}{c}
-k_{2}+\frac{k_{1}}{\left(\left\|\delta \mathbf{x}_{+n}\right\|-x_{\mathrm{DZ}}\right)^{3}} \\
-\frac{\left(\left\|\delta \mathbf{x}_{+n}\right\|-x_{\mathrm{DZ}}\right)^{2}}{2 \varepsilon} \\
0
\end{array}\right\}+\left\{\begin{array}{c}
-k_{2}+\frac{k_{1}}{\left(\left\|\delta \mathbf{x}_{-n}\right\|-x_{\mathrm{DZ}}\right)^{3}} \\
-\frac{\left(\left\|\delta \mathbf{x}_{-n}\right\|-x_{\mathrm{DZ}}\right)^{2}}{2 \varepsilon} \\
0
\end{array}\right\}
\end{gathered}
$$

One can use the same equations of motion (54-59), this time substituting Eqs. (68-71) in turn through Eqs. (37) and performing the same replacements. We now also use, with $\overline{g_{\text {vel }}}, k>0$,

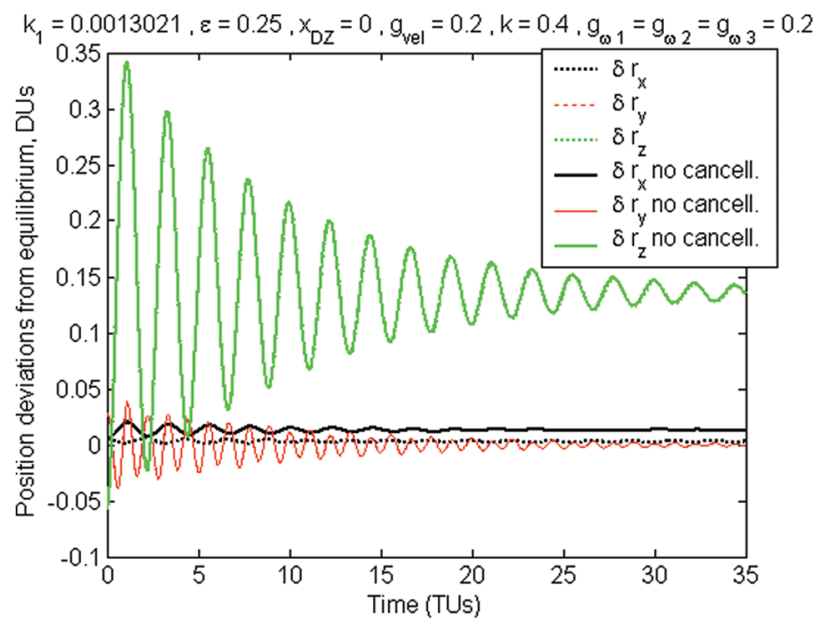

a) Perturbation of position of NEO centroid from that desired for down-track alignment relative equi-librium, coordinated within the GT-fixed frame

Fig. 9 Nonlinear normalized system state responses for three-point-mass bar GT representation with triaxial ellipsoid NEO.

$$
\begin{aligned}
& \mathbf{f}_{c}=\frac{\partial \bar{u}}{\partial \mathbf{r}}+P^{T}\left\{\begin{array}{c}
\frac{-3 k_{1}}{\left(\left\|\delta \mathbf{x}_{-n}\right\|-x_{\mathrm{DZ}}\right)^{5}} \\
-\frac{1}{\varepsilon} \\
0
\end{array}\right\}\left(\delta \mathbf{x}_{-n}-\frac{x_{\mathrm{DZ}} \delta \mathbf{x}_{-n}}{\left\|\delta \mathbf{x}_{-n}\right\|}\right) \\
& +P^{T}\left\{\begin{array}{c}
\frac{-3 k_{1}}{\left(\left\|\delta \mathbf{x}_{+n}\right\|-x_{\mathrm{DZ}}\right)^{5}} \\
-\frac{1}{\varepsilon} \\
0
\end{array}\right\}\left(\delta \mathbf{x}_{+n}-\frac{x_{\mathrm{DZ}} \delta \mathbf{x}_{+n}}{\left\|\delta \mathbf{x}_{+n}\right\|}\right) \\
& +g_{\mathrm{vel}} \mathbf{r}^{\prime}-\left[\begin{array}{c}
0 \\
k g_{\omega 3} r_{d} \delta \omega_{1_{z}} \\
0
\end{array}\right] \\
& \mathbf{m}_{c}=v\left\{\tilde{\mathbf{r}} \frac{\partial \bar{u}}{\partial \mathbf{r}}+\tilde{\alpha}_{T} \frac{\partial \bar{u}}{\partial \alpha_{T}}+\tilde{\beta}_{T} \frac{\partial \bar{u}}{\partial \beta_{T}}+\tilde{\gamma}_{T} \frac{\partial \bar{u}}{\partial \gamma_{T}}\right\} \\
& -\frac{1}{2} \hat{\mathbf{j}} P^{T}\left\{\begin{array}{c}
\frac{-3 k_{1}}{\left(\left\|\delta \mathbf{x}_{-n}\right\|-x_{\mathrm{DZ}}\right)^{5}} \\
-\frac{1}{\varepsilon} \\
0
\end{array}\right\}\left(\delta \mathbf{x}_{-n}-\frac{x_{\mathrm{DZ}} \delta \mathbf{x}_{-n}}{\left\|\delta \mathbf{x}_{-n}\right\|}\right) \\
& +\frac{1}{2} \hat{\mathbf{j}} P^{T}\left\{\begin{array}{c}
\frac{-3 k_{1}}{\left(\left\|\delta \mathbf{x}_{+n}\right\|-x_{\mathrm{DZ}}\right)^{5}} \\
-\frac{1}{\varepsilon} \\
0
\end{array}\right\}\left(\delta \mathbf{x}_{+n}-\frac{x_{\mathrm{DZ}} \delta \mathbf{x}_{+n}}{\left\|\delta \mathbf{x}_{+n}\right\|}\right) \\
& +\left[\begin{array}{c}
-g_{\omega 1} I_{1_{x x}} \delta \omega_{1_{x}}+n_{\text {sun }}\left(I_{1_{z z}}-I_{1_{y y}}\right) \delta \omega_{1_{y}} \\
n_{\text {sun }}\left(I_{1_{x x}}-I_{1_{z z}}\right) \delta \omega_{1_{x}}-g_{\omega 2} I_{1_{y y}} \delta \omega_{1_{y}} \\
-g_{\omega 3} I_{1_{z z}} \delta \omega_{1_{z}}
\end{array}\right]
\end{aligned}
$$

this time with Eqs. (68-72) substituted and the replacements made.

The sketch of the proof of local Lyapunov stability of the equilibrium point, again temporarily assuming $n_{\text {sun }}$ and perfect cancellation of the natural potential with the control, is exactly the same for this case of the bar GT as for the case of the pendulum GT, except that we use the preceding new control force and moment in the equations of motion, and in the domain and Lyapunov function definitions, we have all instances of $\delta \mathbf{x}_{b}$ replaced by $\delta \mathbf{x}_{-n}$ and all instances of $\delta \mathbf{x}_{h}$ replaced by $\delta \mathbf{x}_{+n}$.

Analogous to the previously shown results, Figs. $\underline{9}$ and 10 show the response to the same initial conditions and parameter space values used in the simulations of the last subsection, but for the current three-point-mass bar GT configuration paired with both the

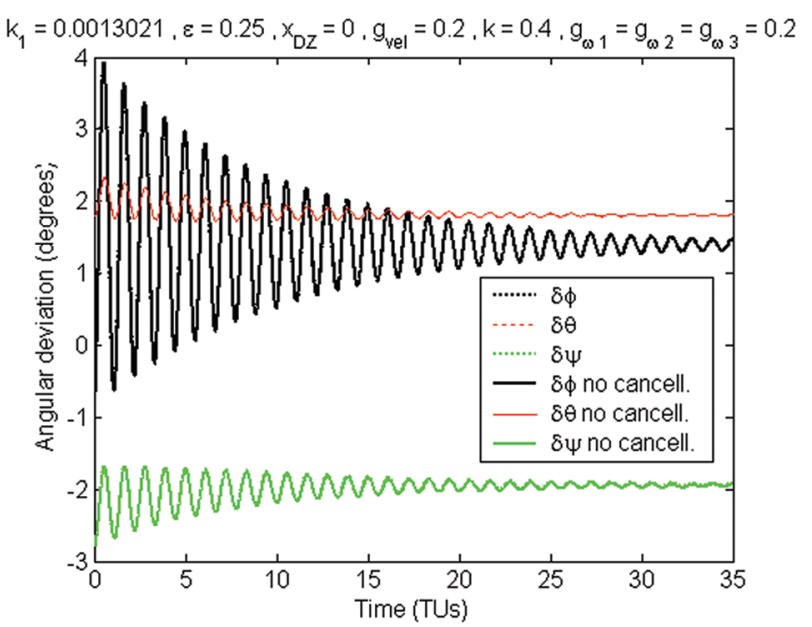

b) Deviation of relative attitude angles from desired relative attitude angles for down-track alignment relative equilibrium 


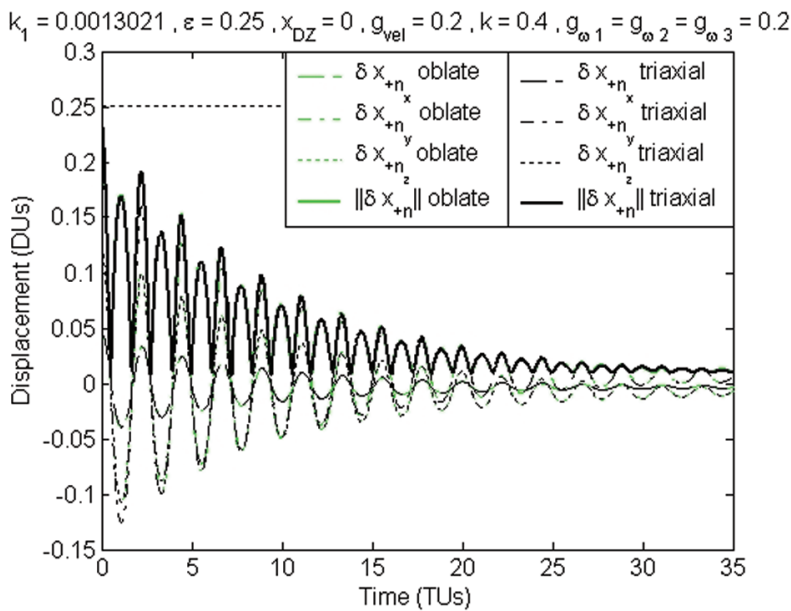

a) $+\hat{\mathbf{j}}$ axis thruster node

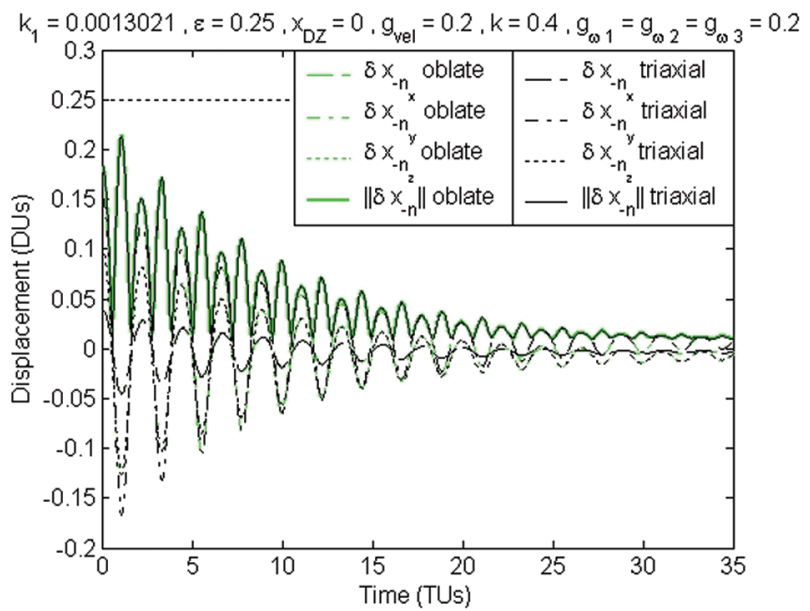

b)- $\hat{j}$ axis thruster node

Fig. 10 Nonlinear normalized system response in position error of the GT nodes from their desired position in the inertial frame fixed to the NEO spin axis; with no cancellation of natural potential for the downtrack alignment relative equilibrium and for the three-point-mass bar GT paired with both oblate spheroid NEO and triaxial ellipsoid NEO.

oblate NEO and the ellipsoid NEO (in Fig. 10) or just the latter (in Fig. 9). The ellipsoid NEO again is rotating with a period of $2 \mathrm{~h}$ about its smallest semi-axis. The gains are altered again for this configuration, with respect to previously shown cases. The excitations from the time-varying mutual gravity in this case are again adequately absorbed with our control. The inertial displacement of each mass in the GT body from its desired precessing (for downtrack alignment) equilibrium position is again effectively eliminated for this novel GT configuration.

Our development of each control approach presented up to this point generally assumes that we have full observability for the states of the final equations of motion in each case. The possible sources of error in measuring these states are larger for some states than for the rest, the most uncertain ones being the components of $\delta \mathbf{r}$, deviations from the desired relative position vector from the GT centroid to the NEO centroid. The exact position of the latter will be highly uncertain until constrained by a significant amount of optical navigation data, such as surface images processed with stereophotoclinometry, lidar mapping, and so on. Measurement of the velocity and angular velocity deviations $\left(\delta \mathbf{r}^{\prime}, \mathbf{r}^{\prime}, \delta \omega_{1}\right)$ should be comparatively easier, employing gyros and lidar information together with usual range and Doppler methods as allowed by lineof-sight access to Earth (though that is potentially quite limited once in close proximity to the target NEO). Measurement of the attitude deviations $\left(\delta \phi\right.$, etc.) from the desired attitude angles $\left(\phi_{d}\right.$, etc.) using star cameras is likely to have the lowest uncertainty. Given the highlevel and approximate nature of the GT designs presented in this paper, a detailed analysis of navigation sensor systems and sensitivity to their error levels is beyond the scope of this article.

\section{Detailed Numerical Simulations Using Developed Control Laws}

Next, we examine four test scenarios for deflection of 99942 Apophis and 1448982004 VD17 using pendulum and bar GT designs. These two NEOs were selected because they are in the current set of known asteroids with nonzero probability of Earth impact, even with significant amounts of tracking data available [12]. They have also been previously examined for deflection using GTs [2]. Table 1 shows the heliocentric orbit elements for these two NEOs. Of the physical properties of these bodies also included in Table 1 , most are still undetermined. So we make best guesses or guesses that place the greatest demands on our control architecture and assign parameter values accordingly. The specific parameter values thus chosen are unrepresentative of our current lack of knowledge concerning these bodies, but are still useful for a point study. All semi-axis distances listed are used exactly in the analytical ellipsoid NEO models, but only approximated by the polyhedral mesh NEO models. Those are shown in Fig. 11 and include artificially produced shape asymmetries and irregularities, to demonstrate robustness to greater perturbations.

We also select the geometry and various parameters for both the pendulum and bar GT spacecraft, as summarized in Table 2. These parameters match with the detailed polyhedral models shown in Figs. $\underline{1}$ and $\underline{2}$. As applicable, these parameters can be used for the corresponding point-mass models as well. We also show the value of the nominal hovering distance $r_{d}$ that defines, along with the initial desired angles and $n_{\text {sun }}$ value of Table 1 , the desired point about which the states deviate at any given timé.

Table 1 Orbit elements and parameters (hypothetical, unless otherwise indicated) of target NEOs used in detailed numerical simulation scenarios

\begin{tabular}{|c|c|c|}
\hline Parameter & 99942 Apophis $\underline{\text { }}$ & 144898 2004VD17 \\
\hline Mean diameter, $\mathrm{m}-$ & 250 & 580 \\
\hline$a, \mathrm{~m}$ & 170 e & 340 \\
\hline$b, \mathrm{~m}$ & $120 \mathrm{e}$ & 280 \\
\hline$c, \mathrm{~m}$ & $96 \mathrm{e}$ & 255 \\
\hline Density, $\mathrm{kg} / \mathrm{m}^{3}$ a & 2600 & 2600 \\
\hline Total mass, kga & $2.1 \times 10^{10}$ & $2.6 \times 10^{11}$ \\
\hline$T_{\text {rot }}, \mathrm{h}$ & $16.0^{\mathrm{f}}$ & 2.0 \\
\hline$\phi_{d}(0), \operatorname{deg}$ & 80 & 15 \\
\hline$\theta_{d}(0), \mathrm{deg}$ & 10 & 50 \\
\hline$\psi_{d}(0), \operatorname{deg}$ & 45 & 45 \\
\hline$a, \mathrm{AU} \cong$ & 0.92226 & 1.50812 \\
\hline$e^{\mathrm{a}}$ & 0.191059 & 0.588704 \\
\hline$i, \operatorname{deg} \mathrm{a}$ & 3.33131 & 4.22293 \\
\hline Perihelion, AUa & 0.74605 & 0.62028 \\
\hline Aphelion, AU & 1.09847 & 2.39595 \\
\hline$\omega$, dega & 126.39 & 90.686 \\
\hline$\Omega$, deg $\underline{\mathrm{a}}$ & 204.46 & 224.24 \\
\hline$T_{\text {sun }}$, year- & 0.8857 & 1.8521 \\
\hline$n_{\text {sun }}$ avg, deg / daya & 1.112815 & 0.532171 \\
\hline$n_{\text {sun }}$, deg / day & 1.404736 & 1.604395 \\
\hline
\end{tabular}

abtained from [12], as of 17 January 2007.

${ }^{\mathrm{b}}$ Actual data values indicated are based on 2 radar-delay, 5 Doppler, and 731 optical observations spanning 884.52 days [12]].

${ }^{\mathrm{c}}$ Actual data values indicated are based on 905 observations spanning 1764.9 days [12]. ${ }^{\mathrm{d}}$ This is the maximum rate of change in the downtrack direction, occurring at perihelion of the respective NEO, as calculated from the orbit.

${ }^{\mathrm{e}}$ Following values used in [13]

${ }^{\mathrm{f}}$ From [14]. 


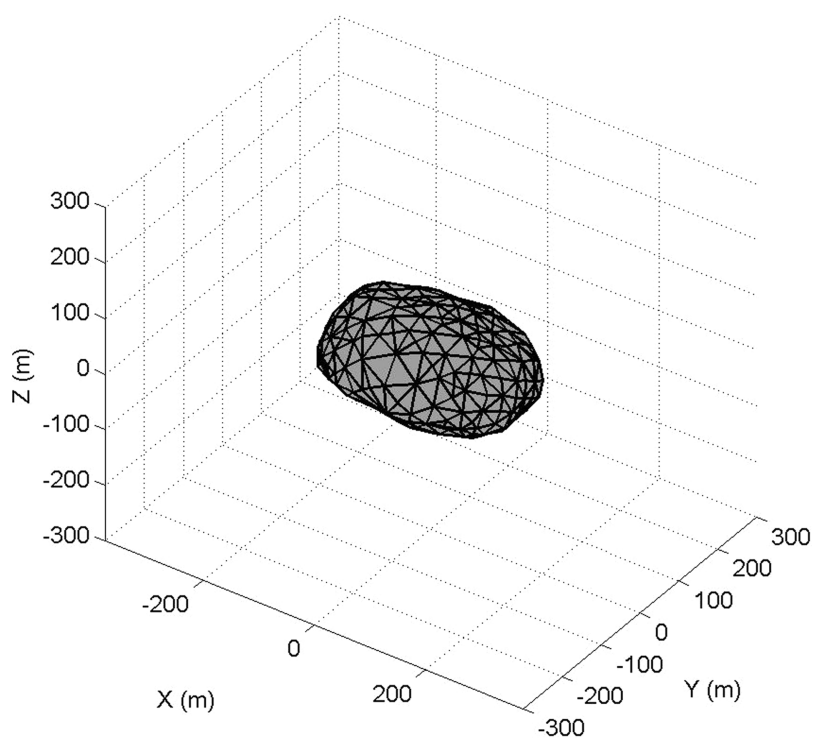

a) 99942 Apophis

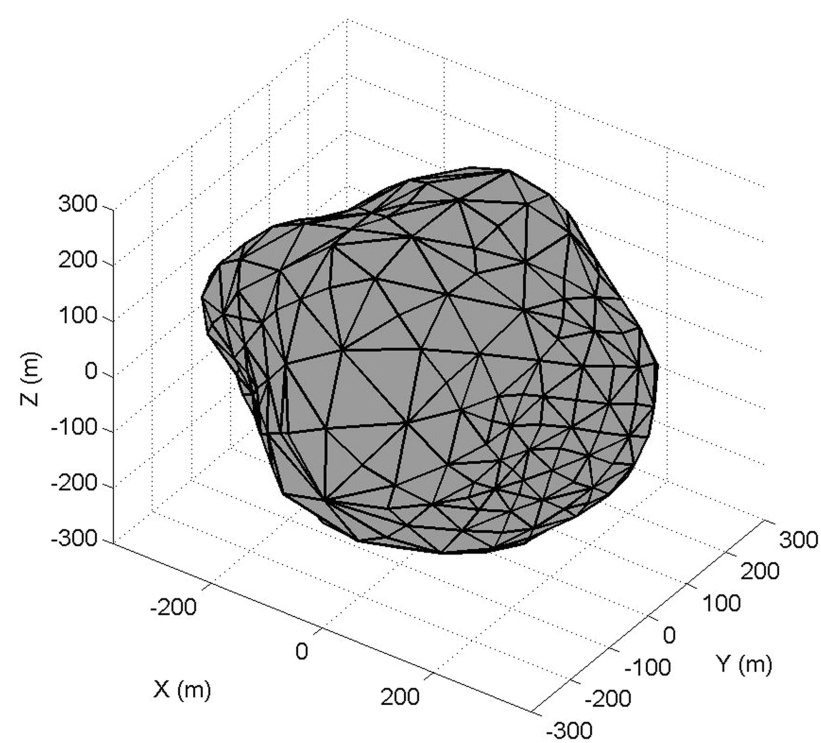

b) 1448982004 VD17

Fig. 11 Illustration of the hypothetical polyhedral body models of NEOs selected for use in the detailed numerical simulation test scenarios. The scale is the same between the two illustrations. It is emphasized that these are purely speculative body models, with no relation to currently limited or nonexistent shape data for these specific asteroids.

Table 3 shows the initial conditions to which the response is obtained using both the analytical models and the full-detail polyhedral models in all four test scenarios. The states to which these initial conditions are applied are propagated directly using the full nonlinear equations (54-59) for the analytical models. For the polyhedral models, which are instead propagated according to the full two-rigid-body-problem methodology outlined in [10-15], this set of states and their corresponding initial conditions are transformed to the set of states used within that methodology. The initial conditions in Table $\underline{3}$ are selected based in part on anticipated $1 \sigma$ measurement uncertainties of $1.0 \mathrm{~m}$ and $1.0 \mathrm{~mm} / \mathrm{s}$, respectively, in the position of each controlled GT node relative to its desired inertialframe position and in the velocity of each controlled node relative to that inertial frame. By controlled node, we mean one having an artificial potential well placed about its desired position. These

Table 2 Parameters of the two types of GTs and their controllers for each of the detailed numerical simulation test scenarios

\begin{tabular}{lcccc}
\hline \hline & \multicolumn{2}{c}{99942 Apophis } & \multicolumn{2}{c}{144898 2004VD17 } \\
\cline { 2 - 5 } Parameter & Pendulum GT & Bar GT & Pendulum GT & Bar GT \\
\hline$r_{d}$ & 5.1 & 5.1 & 15.64 & 15.64 \\
$l, \mathrm{~m}$ & 50 & 50 & 50 & 50 \\
$\eta_{(p, b)}, \mathrm{deg}$ & 55.5 & 56.0 & 44.5 & 43.9 \\
$\mu$ & 0.1128 & 0.1134 & 0.344 & 0.320 \\
$k_{1}$ & 0.00231 & 0.02083 & $6.510 \times 10^{-5}$ & 0.02083 \\
$\varepsilon$ & 4.5002 & 0.5 & 5.000 & 0.5 \\
$x_{\text {DZ }}$ & 0.0 & $1 \times 10^{-10}$ & 0.25 & $1 \times 10^{-10}$ \\
$g_{\omega 1}$ & 10 & 10 & 10 & 10 \\
$g_{\omega 3}$ & 10 & 10 & 10 & 10 \\
$g_{\omega 3}$ & 10 & 10 & 10 & 10 \\
$g_{\text {vel }}$ & 10 & 10 & 10 & 10 \\
$k$ & $\mathrm{n} / \mathrm{a}$ & 20 & $\mathrm{n} / \mathrm{a}$ & 20 \\
$\mathrm{GT} \mathrm{mass}, \mathrm{kg}$ & $2.000 \times 10^{4}$ & $2.000 \times 10^{4}$ & $2.692 \times 10^{4}$ & $3.099 \times 10^{4}$ \\
$I_{1_{1}}, \mathrm{~kg} \mathrm{~m}^{2}$ & $5.323 \times 10^{4}$ & $1.788 \times 10^{6}$ & $7.18 \times 10^{4}$ & $6.485 \times 10^{6}$ \\
$I_{1_{y}}, \mathrm{~kg} \mathrm{~m}^{2}$ & $5.673 \times 10^{6}$ & $6.31 \times 10^{4}$ & $1.57 \times 10^{7}$ & $9.624 \times 10^{4}$ \\
$I_{1_{2}}, \mathrm{~kg} \mathrm{~m}^{2}$ & $5.675 \times 10^{6}$ & $1.801 \times 10^{6}$ & $1.57 \times 10^{7}$ & $6.507 \times 10^{6}$ \\
$r_{b}$ & 0.0482 & 0.0482 & 0.0481 & 0.0511 \\
$h_{b}$ & 0.0963 & 0.0963 & 0.0962 & 0.1021 \\
$r_{(h, n)}$ & 0.0281 & 0.0328 & 0.0366 & 0.0467 \\
$h_{(h, n)}$ & 0.0200 & 0.0200 & 0.0332 & 0.0200 \\
$\eta_{1 / 2}, \mathrm{deg}$ & 20 & 20 & 20 & 20 \\
\hline \hline
\end{tabular}

uncertainties can be mapped from the $\left(\delta \mathbf{x}_{i}, \delta \mathbf{x}_{i}^{\prime}\right)$ space back to the $\left(\delta \mathbf{r}, \mathbf{r}^{\prime}, \delta \boldsymbol{\omega}_{1}, \delta \phi, \delta \theta, \delta \psi\right)$ space with a state-dependent mapping. Actual measurement noise and any filter of it to supply values to the controller inputs are not implemented for the results in this paper.

For each scenario, we plot the norm of the position error (with respect to the desired equilibrium position) vector for both controlled nodes (bottom and head for the pendulum, left and right thruster modules for the bar), with concurrent curves from propagating the analytical models and from propagating the truth polyhedral body models. Figure 12 shows just that for the deflection of Apophis using the pendulum GT configuration, the deflection of Apophis with the bar GT configuration, deflection of 2004 VD17 with the pendulum GT, and deflection of the same with the bar GT.

It is seen that over the full five NEO rotation periods for Apophis or 50 NEO rotation periods for VD17, adequate performance and trajectory behavior is achieved for both models with the use of the tabulated controller parameters. The amplitudes of oscillations persisting in the steady state are larger for the polyhedral models than for the analytical ones, as expected once the full complexity of the body shapes is included. The details of how the higher-order mass distribution excites and maintains the additional oscillations may merit further investigation. The steady-state bias for both models is in the general direction of the NEO and nearly the same between models, also as expected, although there is a further bias of the pendulum at VD17 using the polyhedra. Two additional features of the responses are noted that do not appear in Fig. 12. First, in each scenario, the assumption that the NEO has clean principal-axis rotation without free precession and nutation does not hold exactly using the polyhedral models. No special effort was made to realign the mesh approximating the ellipsoid NEO to ensure that the new principal axis that mesh has (consistent with its asymmetry and

Table 3 Initial conditions for states of system (all in normalized units)

\begin{tabular}{lccc}
\hline \hline State/quantity & Initial condition & State/quantity & Initial condition \\
\hline$\delta \mathbf{r}_{x}$ & -0.048 & $\delta \omega_{1_{x}}$ & -0.1902 \\
$\delta \mathbf{r}_{y}$ & 0.155 & $\delta \omega_{1_{y}}$ & 0.0443 \\
$\delta \mathbf{r}_{z}$ & -0.337 & $\delta \omega_{1_{z}}$ & -0.0402 \\
$\mathbf{r}_{x}^{\prime}$ & 0.0245 & $\delta \phi, \mathrm{rad}$ & $5.24 \times 10^{-5}$ \\
$\mathbf{r}_{y}^{\prime}$ & -0.0209 & $\delta \theta, \mathrm{rad}$ & $-5.24 \times 10^{-5}$ \\
$\mathbf{r}_{z}^{\prime}$ & -0.0384 & $\delta \psi, \mathrm{rad}$ & $-5.24 \times 10^{-5}$ \\
\hline \hline
\end{tabular}




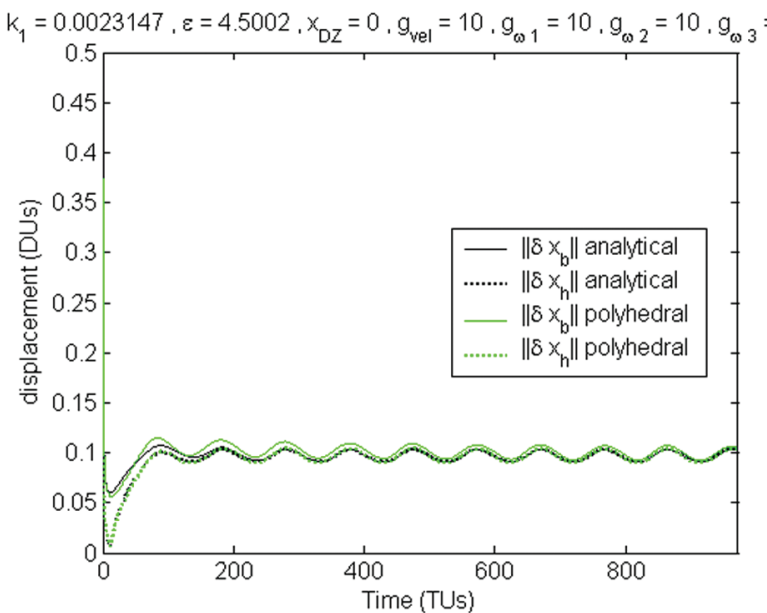

a) For deflection of 99942 Apophis by pendulum GT

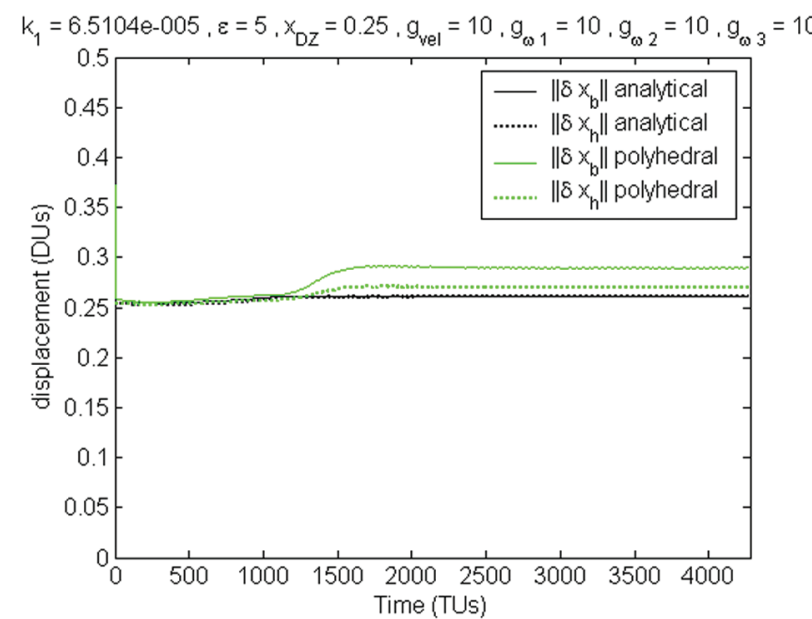

c) For deflection of 1448982004 VD17 by pendulum GT

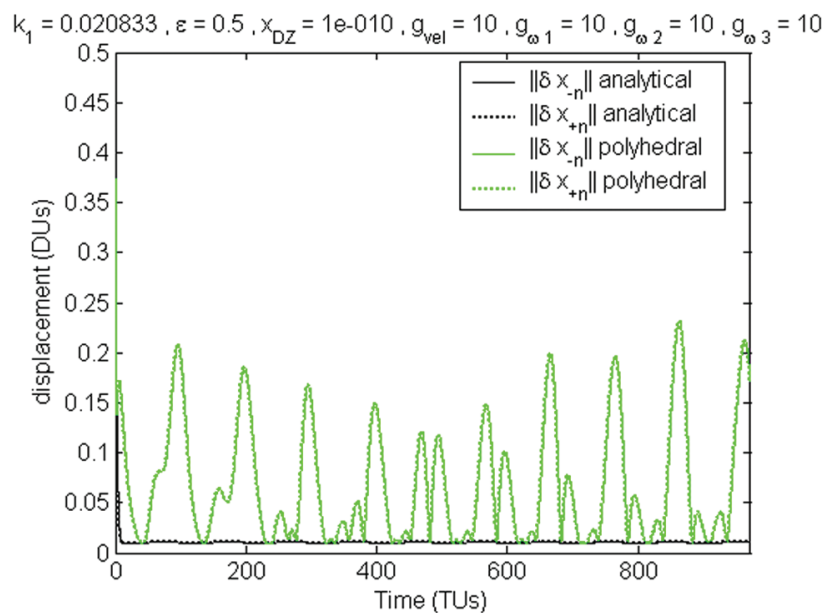

b) For deflection of 99942 Apophis by bar GT,

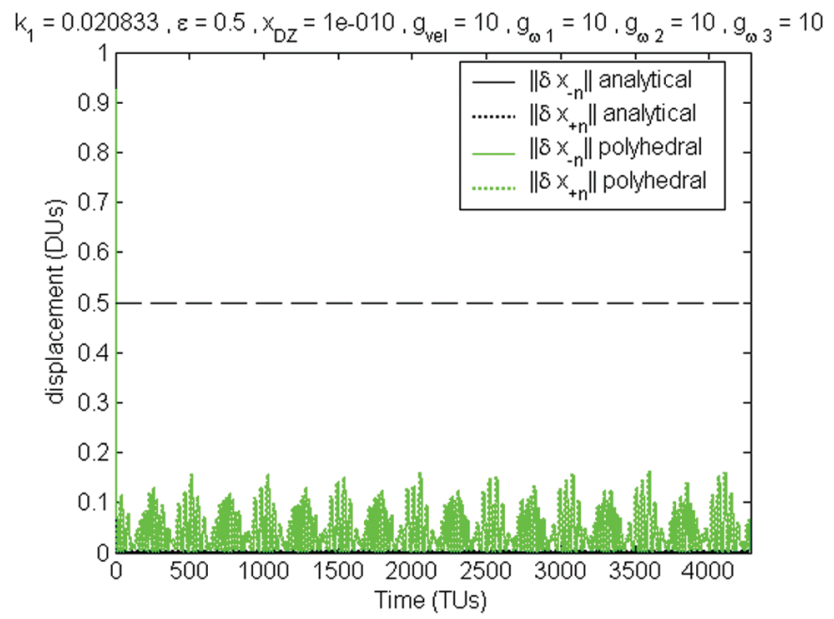

d) For deflection of 1448982004 VD17 by bar GT

Fig. 12 Norm of vector position errors of controlled nodes with respect to their desired positions for the downtrack alignment relative equilibrium, coordinated in the frame fixed to the NEO in each case.

detailed shape) is parallel with the initial-time spin axis. The resulting nontrivial polyhedral NEO rotation is more severe (maximum nutation angle of $6 \mathrm{deg}$ ) with our hypothetical VD17 mesh than for our Apophis mesh. Second, for scenarios using a bar GT, there can be large rotation oscillations about the boom centerline that persist only for the polyhedral models, indicating that a much stronger $g_{\omega 2}$ gain is needed going from the analytical to the polyhedral models, to handle the detailed GT mesh's asymmetry about its boom centerline.

\section{System Design, Performance, and Implications}

We seek to analyze and quantify any performance penalty that is incurred by using the appropriate developed controller, for each combination of deflection target and GT type in the last section. In each case, we seek to describe such performance over a sensible set of system parameters. The specific target NEO's chosen properties lock in many parameters at the start. Even so, describing performance over the whole remaining parameter space is intractable. Thus, we restrict ourselves to exploring the lower-dimension parameter subspace formed by 1) the length $l$ from the center of mass of the bottom to the center of mass of the head, or between the centers of mass of the outboard thruster nodes; 2) the total GT mass $m_{1} ; 3$ ) the GT internal mass fraction $\mu ; 4)$ the various control gains $g_{\text {vel }}$ and $g_{\omega_{i}}$ (and, as applicable, $k$ ); and 5) the well shape and size parameters $k_{1}$ and $\varepsilon$ and the dead-zone distance $x_{\mathrm{DZ}}$, as shown in Fig. 6 .

It is possible to further reduce this parameter subspace. Additional constraint relations can determine both $m_{1}$ and $\mu$ indirectly from $l$ and from the parameter values fixed at the start and thus not listed. As in [1], we always assume that the thruster exhaust plumes from the main engines of both bar and pendulum GTs remain exterior to the sphere of radius $R_{\text {sph }}$ (in meters) circumscribing the NEO, to avoid ever impinging on the NEO surface. The bore sights of opposing engine pairs must therefore be canted away from the towingdirection vector by a minimum angle $\eta_{p}$ or $\eta_{b}$ (for pendulum or bar GT, respectively). This angle is determined through geometry from the engine exhaust plume half-angle $\eta_{1 / 2}$ and the values for $R_{\mathrm{sph}}, r_{d}$, and $l$ (as well as $\mu$ for the pendulum only). The first-cut idealized spherical NEO plus single-point-mass GT setup appearing in previously published work (hereafter, the baseline) give a first value for the net force that the engines must apply to the GT for open-loop hovering within that setup. The total engine thrust capacity needed can be first estimated by dividing this force by the cosine of $\eta_{(p, b)}$. Through very rough design of a nuclear electric propulsion system to supply this engine thrust capacity plus some margin, masses for the elements of the GT assumed to form the main body (e.g., reactor) and the head or outboard nodes (e.g., engines, propellant, and radiators) are determined. This gives a total GT mass and how it is split between the main body and head/nodes (i.e., values for $m_{1}$ and $\mu$ ). Because the original hovering force and GT placement geometry generally change with a change to $m_{1}$ or $\mu$, this rough-design process is iterated to convergence. Interestingly, we find that system design does not allow just any arbitrary $\mu$, and explosive growth in total spacecraft mass results for particularly poor placements of the GT. We do not go into these system design issues further, because our focus is on the dynamics and control of the GT. The key idea is that we use system 
design to specify final converged $m_{1}$ and $\mu$ values, along with the moments of inertia, etc., eliminating them from the list of still-free parameters.

Some constraints can also be placed on the well shape and size parameters. We do not desire to allow a controlled node of the GT to move outside of the outermost transition radius at which a switch between pieces of the piecewise well function occurs. Because of weakening restoring force with larger deviation once past this transition radius, as soon as a node crosses it going away from the equilibrium point, that node is unlikely to return. Between the deadzone boundary and this transition radius, the restoring force and control effort needed are inversely proportional to $\varepsilon$, and so to reduce control effort, we might seek to increase $\varepsilon$ as much as possible without causing the controller to fail. Here, failure is defined by allowing any node to cross the outer transition radius of its well within a simulation time sufficient to cover several NEO rotation periods. However, because that radius is itself dependent on $k_{1}$ in addition to $\varepsilon$, one could independently choose $k_{1}$ to make that radius arbitrarily large, and this failure criterion becomes meaningless. Instead, suppose that to ensure no node position reversal (inversion of the pendulum or flipping about the local vertical for the bar), we set the transition radius to 0.5 times the boom length. The value of $k_{1}$ is determined by $\varepsilon$ and $x_{\mathrm{DZ}}$ to satisfy this constraint. As for $\varepsilon$ itself, we choose this small enough to satisfy the bound

$$
\forall \delta \mathbf{x}_{i} \in\left\{\mathbb{B}_{0.5}(0)-\mathbb{B}_{\kappa}(0)\right\}, \quad \frac{1}{\varepsilon}>\frac{\left\|\partial u / \partial \delta \mathbf{x}_{i}\right\|}{\left\|\delta \mathbf{x}_{i}\right\|}
$$

for all $i$-controlled nodes. Here, the inner ball's radius of $\kappa$ should prevent singularity on the right-hand side of the inequality and might be set to $x_{\mathrm{DZ}}$ if that is nonzero. This rule ensures that the gradient due to the potential wells is always stronger than the natural potential gradient at all points between concentric spherical shells with radii of $\kappa$ and 0.5 normalized units, centered on the desired equilibrium point for every GT-controlled node. Except for cases with large initial conditions in velocity states, this provides a near guarantee to not encounter the failure criterion. For a point of reference, setting $\kappa=0.05$ and evaluating this upper bound on $\varepsilon$ for each of the test scenarios of the last section gives approximately 0.98 for Apophis and the pendulum, 1.04 for Apophis and the bar, 11.66 for VD17 and the pendulum, and 12.19 for VD17 and the bar.

To facilitate any further reduction of the parameter subspace, we must obtain the performance measures to use and look at their values at some design points. There are many possible metrics to examine, but in terms of the end goal of the deflection effort, we are interested in total downtrack $\Delta V$ applied to the NEO + GT system as a whole. More specifically, we wish to quantify the reduction of $\Delta V$ achievable using our controller below the $\Delta V$ achievable with the baseline. Such comparison is only valid given a fixed fuel amount. The components of all thruster exhaust plumes in directions normal to the towing-direction vector may be assumed to apply no downtrack $\Delta V$ to the system. Components of thruster exhaust plumes in the same direction as the towing-direction vector directly reduce that $\Delta V$ as well. Use of our control scheme may require thruster output in these directions greater (lesser) than the thruster output in those directions with the baseline. If so, then for a fixed fuel quantity, the total $\Delta V$ is proportionately reduced (increased). Equivalently, the total time that the deflection operation can be continued before exhausting fuel is reduced (increased).

To eliminate the time of operation and fuel amounts from the performance analysis, one can shift to performance metrics in terms of accelerations or forces. From this perspective, the benefit from GT operation can be measured as the component of the acceleration or force exerted on the NEO that is parallel to the desired towing direction, averaged over time. This can be represented by

$$
f_{\text {ben }}=\frac{1}{t_{f}-t_{o}} \int_{t_{o}}^{t_{f}}\left(-\mathbf{f}_{1} \cdot \hat{\mathbf{w}}\right) \mathrm{d} \tau
$$

where $\hat{\mathbf{w}}$ is the unit vector aligned to the desired towing direction at all times, and $t_{o}$ and $t_{f}$ are the start and end times of the simulation. They should be separated by the greater of at least several NEO periods of rotation or the characteristic timescale observed for reaching an approximate steady state. Of course, the corresponding cost from GT operation is not as easily computed. Not wishing to delve into thruster layout and thruster mixing logic, we combine the net control thrust and moment used in the propagated equations into one number for the total thruster effort required, which we average over the simulation time as well.

The approach is as follows: First assume all control moments are provided by the action of thrusters (very conservative) and that the moment component about the longest axis of the craft is provided by equal and opposite thrusts applied at the body points, with the largest moment arm about that axis $\left(r_{b}\right.$ and $h_{b} / 2$ for the pendulum and bar, respectively). The remaining moment components are provided by equal and opposite thrusts applied at the extreme ends of the craft, for the largest possible moment arm. In the desired equilibrium attitude, all of these thrusts for control moment are perpendicular to the towing direction and represent lost thruster effort. Take any positive projection of the required control force $\mathbf{f}_{c}$ onto the GT-fixed frame's $\hat{\mathbf{i}}$ vector and assume this is provided by main engines and hence subject to the $1 / \cos \left(\eta_{(p, b)}\right)$ engine cant penalty. The norm of the remainder of the $\mathbf{f}_{c}$ vector, or all of it if it has no positive projection onto $\hat{\mathbf{i}}$, is counted as additional lost thruster effort. By summation, we have the following expressions for time-averaged thruster-effort "cost" for the pendulum and bar GTs, respectively:

$$
\begin{aligned}
f_{\text {cost }} & =\frac{1}{t_{f}-t_{o}} \int_{t_{o}}^{t_{f}}\left[\frac{\left|\mathbf{m}_{c_{x}}\right|}{r_{b}}+2 \sqrt{\mathbf{m}_{c_{y}}^{2}+\mathbf{m}_{c_{z}}^{2}}+\frac{\max \left\{0, \mathbf{f}_{c} \cdot \hat{\mathbf{i}}\right\}}{\cos \left(\eta_{p}\right)}\right. \\
& \left.+\left\|\mathbf{f}_{c}-\max \left\{0, \mathbf{f}_{c} \cdot \hat{\mathbf{i}}\right\} \hat{\mathbf{i}}\right\|\right] \mathrm{d} \tau \\
f_{\text {cost }} & =\frac{1}{t_{f}-t_{o}} \int_{t_{o}}^{t_{f}}\left[\frac{2\left|\mathbf{m}_{c_{y}}\right|}{h_{b}}+2 \sqrt{\mathbf{m}_{c_{x}}^{2}+\mathbf{m}_{c_{z}}^{2}}+\frac{\max \left\{0, \mathbf{f}_{c} \cdot \hat{\mathbf{i}}\right\}}{\cos \left(\eta_{b}\right)}\right. \\
& \left.+\left\|\mathbf{f}_{c}-\max \left\{0, \mathbf{f}_{c} \cdot \hat{\mathbf{i}}\right\} \hat{\mathbf{i}}\right\|\right] \mathrm{d} \tau
\end{aligned}
$$

The cost/benefit ratio $f_{\text {sys }}=f_{\text {cost }} / f_{\text {ben }}$ for our systems using the controller schemes presented herein can be compared with the cost/ benefit ratio $f_{\text {basic }}=1 / \cos \left(\eta_{(p, b)}\right)$ for the corresponding baseline. The ratio of $f_{\text {sys }}$ over $f_{\text {basic }}$ gives a scalar comparative performance degradation factor $f$. All requirements worked out very quickly with the baseline setup for system thrust, power, and fuel capacity must be at least multiplied by the factor $f$ to yield minimum requirements for the actual GT design if that design will achieve the same desired towing effect as the baseline. This is a general quantity, dictated for each target-body and GT-type combination by the natural full tworigid-body-problem dynamics of that combination and the remaining free parameters. As for those remaining parameters $\left(l, g_{\text {vel }}, g_{\omega_{i}}, k, \varepsilon, x_{\mathrm{DZ}}\right)$, conducting sparse manual exploration of the values of $f_{\text {ben }}$ through $f$ at different points by simulating the response to the initial conditions of Table 3 over several NEO periods reveals a few trends.

First, one should include a fairly high level of damping. Without this, large position and velocity oscillations persist over time. Even though they may have amplitude well below that sufficient to violate the 0.5 bound on node position deviation, the periodic controller thrusting that produces the oscillation increases $f_{\text {cost }}$ greatly, requiring unacceptably large $f_{\text {sys }}$ values and infeasible system design. Hence, we lock the gains $g_{\text {vel }}, g_{\omega_{i}}$, and $k$ to values on the order of 10 .

Second, with the nominal hovering distance $r_{d}$ fixed, the force required by the baseline for hovering of the GT remains the same. Then increasing the boom length $l$ tends to decrease the minimum engine cant angles $\eta_{(p, b)}$. With the pendulum, there is the additional effect of bringing the bottom closer to the NEO with larger $l$. Hence, with increasing $l$, the towing effect and $f_{\text {ben }}$ tend to stay the same (or increase), but both $f_{\text {basic }}$ and $f_{\text {sys }}$ tend to decrease (mainly through the cant angles). However, we observe that the former decreases faster, 
such that $f$ grows slightly, with increasing $l$. This could be misleading about the overall cost/benefit of such an extension of the spacecraft, but we view absolute improvement in overall towing capability represented by lower $f_{\text {sys }}$ as far more important than the comparative performance difference between the baseline and actual simulated models. This means an advantage to extending the GT boom as long as possible, up to the limit of feasibility for in-space construction or to the point at which assuming a rigid GT body becomes problematic. Hence, we do not search for the minimum of any metric along $l$, but lock in the large, but feasible, value of $l=50 \mathrm{~m}$.

Related to selecting a boom length, note that whether the novel bar configuration introduced in this paper performs better or worse than the pendulum configuration for deflecting a specific NEO, with all parameters common to the two configurations being equal, is answered differently for different ranges of $l$. Assume we set values of $r_{d}$ such that, between both configurations, we maintain the same safety margin of $s$ (in meters) between the surface of the NEO circumscribing sphere and the centroid of the main body of the GT. Perhaps for the greatest value of $f_{\text {ben }}$, we will want to reduce $s$ as small as can be safely allowed, though our exploration suggests that would likely lead to divergence in GT mass under iterative system design. In any case, $r_{d}=\mu+\left(R_{\text {sph }}+s\right) / l$ for the pendulum and $r_{d}=\left(R_{\mathrm{sph}}+s\right) / l$ for the bar. Supposing the same $\eta_{1 / 2}$ and the same $l$, the geometry dictates that

$$
\eta_{p}=\sin ^{-1}\left(\frac{R_{\mathrm{sph}}}{R_{\mathrm{sph}}+s+l}\right)+\eta_{1 / 2}
$$

$$
\begin{aligned}
\eta_{b} & =\sin ^{-1}\left(\frac{R_{\mathrm{sph}}}{\sqrt{\frac{l^{2}}{4}+\left(R_{\mathrm{sph}}+s\right)^{2}}}\right) \\
& -\sin ^{-1}\left(\frac{l}{2 \sqrt{\frac{l^{2}}{4}+\left(R_{\mathrm{sph}}+s\right)^{2}}}\right)+\eta_{1 / 2}
\end{aligned}
$$

The value of $l$ as a function of $R_{\mathrm{sph}}$ and $s$, for which these angles are equal, is the discriminating value. When $l$ is longer, the bar produces the same open-loop hovering thrust of the baseline, with a lower $f_{\text {basic }}$ than the pendulum, so that the bar configuration is favored before applying our $f$ factor. When $l$ is shorter, the reverse holds, so that the pendulum configuration is so favored. Put another way, if $f$ were about the same for both configurations, then all other things being equal, our hypothesis that the bar configuration allows a lower $f_{\text {sys }}$ than the pendulum configuration would tend to be true for $l$ longer than this discriminating value, but false for $l$ shorter than it. In each detailed test scenario of Sec. IV, the $l=50 \mathrm{~m}$ chosen is quite small compared with the NEO largest semi-axis, so it is not surprising to observe that in most of these cases, after applying the $f$ to the $f_{\text {basic }}$, the pendulum performs better with lower $f_{\text {sys }}$. However, $l=50 \mathrm{~m}$ is above the calculated discriminating value for both VD17 setups and below that value for both Apophis setups, and so it is apparent that a highly variable $f$ can override the previous general statements. Finally, note that only the bar configuration with the very large $l$ value of

$$
l \geq 2\left(R_{\mathrm{sph}}+s\right) \tan \left(\eta_{1 / 2}\right)+\frac{2 R_{\mathrm{sph}}}{\cos \left(\eta_{1 / 2}\right)}
$$

gives engine cant angles of zero and hence the smallest possible $f_{\text {basic }}=1$.

For each of the four test scenarios of the last section, we conducted a formal gradient search for the minimum $f_{\text {sys }}$ over the two free parameters now remaining: $\varepsilon$ and $x_{\mathrm{Dz}}$; more specifically, over a patch in quadrant I of the $\varepsilon-x_{\mathrm{DZ}}$ plane, with boundaries chosen in part based on the bounds computed with Eq. (76) for each NEO's natural gravitational potential. For our search, we used a standard optimization package wrapped around comparatively fast simulation of the analytical models, in response to the initial conditions of Table $\underline{3}$, covering five Apophis rotation periods or 50 much shorter VD17 rotation periods, as applicable. We also further detailed sparsely sampled points along the search path through the $\varepsilon-x_{\mathrm{DZ}}$ plane with otherwise identical propagations of the dual polyhedral NEO and GT models using parallel implementation of the exact full two-body problem integration method outlined in [10].

It is observed that starting in the most conservative smallest $\varepsilon$ and smallest $x_{\mathrm{DZ}}$ corner of the search space, after moving only a short distance away from that corner, $f_{\text {sys }}$ becomes relatively flat, although still slightly decreasing with larger $\epsilon$. Thus, for minimal performance improvement, the search path moves all over (and, generally, away) until (even though the analytical model propagation still shows nicely bounded behavior) the polyhedral propagation shows system failure in the face of its greater perturbations. We desired to present results sets for each of the four test scenarios covered in the last section, matching parameter values identified as minimum $f_{\text {sys }}$ points, but instead we have chosen to show results for nonoptimal points not close to being at risk of breaking the system. Both $f_{\text {sys }}$ and the other performance metrics found along with it for each of the four presented results sets are listed in Table 4 ; the preceding tables give the matching underlying points in the parameter space.

The small-to-moderate size of $f$ in these test scenarios suggests that the level of difficulty of controlling the whole system vs uncontrolled operation is manageable, but much less so for the bar GT at VD17 and especially so for the bar GT at Apophis. The relative sizes of the changes in the metrics between the analytical and polyhedral models indicate that it is indeed important to incorporate high-fidelity shape models and all of their perturbing effects, as we do herein.

From the size of the change in all metrics between different target bodies having different characteristics, but given use of the same GT configuration, we also discern a significant need for modeling of target bodies before such a large-scale GT deflection mission is designed and built. By modeling, we mean not just precise orbit determination and gross diameter inference alone, but the best possible determination of size, shape, spin state, and other physical characteristics. This might be done through ground-based planetary radar if there is a suitable observation window or a more costly (but potentially better return) precursor characterization mission. Some prior claims made to the effect that because the GT does not need to physically connect with the NEO, there is no need for prior knowledge of the asteroid's shape, composition, rotation rate, etc., appear to be incorrect.

Table 4 Performance metrics for parameter subspace points having simulation results shown in this paper for each of the detailed numerical simulation test scenarios; the two values in each entry are for the analytical/polyhedral runs

\begin{tabular}{lcccc}
\hline \hline & \multicolumn{2}{c}{99942 Apophis } & \multicolumn{2}{c}{144898 2004VD17 } \\
\cline { 2 - 5 } Perf. metric & Pendulum GT & Bar GT & Pendulum GT & Bar GT \\
\hline$f_{\text {ben }}, \mathrm{N}$ & $0.4885 / 0.4860$ & $0.4654 / 0.4652$ & $0.7975 / 0.7883$ & $0.8835 / 0.8875$ \\
$f_{\text {cost }}, \mathrm{N}$ & $0.8999 / 0.9032$ & $0.9015 / 0.7487$ & $1.1811 / 1.1820$ & $1.8050 / 1.9560$ \\
$f_{\text {sys }}$ & $1.8422 / 1.8583$ & $1.9373 / 1.6093$ & $1.4811 / 1.4995$ & $2.0430 / 2.2038$ \\
$f_{\text {basic }}$ & $1.7671 / 1.7671$ & $1.7868 / 1.7868$ & $1.4023 / 1.4023$ & $1.3884 / 1.3884$ \\
$f$ & $1.0425 / 1.0516$ & $1.0842 / 0.9007$ & $1.0562 / 1.0693$ & $1.4715 / 1.5873$ \\
\hline \hline
\end{tabular}




\section{Conclusions}

For increasingly complex and more realistic models of any impact-threatening NEO, both the pendulum gravity tug and our novel bar gravity tug can be suitably controlled to complete a deflection operation, using the specific controller designs presented in this paper. The desired deflection operation achieved applies continuous change in heliocentric orbital velocity in a direction close to the most desirable precessing downtrack velocity vector (or any inertially fixed direction as a special case). This holds true in the presence of realistic perturbations and the effects of full geometric detail for both the natural and artificial bodies involved. The important performance metrics we have introduced for design of a gravity-tug system having either the pendulum or bar configuration mostly capture the difficulty of controlling to the desired outcome, as driven by the fully coupled rotational and translational dynamics and the system parameters. The effort required is seen to be reasonable for a few point studies. Attempting to rigorously minimize these performance metrics may weaken the controller to the point that the system fails, and so each scenario requires careful examination.

\section{Appendix A: Point-Mass and Ellipsoid Representations of Pendulum GT with Sphere NEO}

First, for the point-mass pendulum GT representation, let the bottom of mass $m_{b}$ (which is the majority of the GT's total mass $m_{1}$ ) and head of mass $m_{h}$ be separated by a boom of normalized length $l$. Introducing the ratio $\mu=m_{h} /\left(m_{b}+m_{h}\right)$, and with our prior alignment of the frame attached to the GT, the key vectors at both desired equilibria are

$$
\begin{gathered}
\mathbf{r}_{\mathrm{eq}}=\left[\begin{array}{lll}
-r_{d} & 0 & 0
\end{array}\right]^{T}, \quad \rho_{b}=\left[\begin{array}{lll}
-\mu l & 0 & 0
\end{array}\right]^{T} \\
\rho_{h}=\left[\begin{array}{lll}
l-\mu l & 0 & 0
\end{array}\right]^{T}
\end{gathered}
$$

Using these within the basic formulation of the mutual potential and its first and second partial derivatives for this case and employing the definition of $\mu$, we can identify

$$
\begin{gathered}
x=-r_{d}, \quad u_{x}=\frac{1-\mu}{\left(r_{d}-\mu l\right)^{2}}+\frac{\mu}{\left(r_{d}+l-\mu l\right)^{2}} \\
u_{x x}=2\left(\frac{1-\mu}{\left(r_{d}-\mu l\right)^{3}}+\frac{\mu}{\left(r_{d}+l-\mu l\right)^{3}}\right) \\
u_{y y}=u_{z z}=-\left(\frac{1-\mu}{\left(r_{d}-\mu l\right)^{3}}+\frac{\mu}{\left(r_{d}+l-\mu l\right)^{3}}\right)
\end{gathered}
$$

For purposes of the inertia dyad elements, one can represent the geometry of the GT with dual uniform-density cylinders and a massless link, as shown in Fig. 1 . This leads to

$$
\begin{gathered}
\mathrm{I}_{1_{x x}}=\frac{1}{2} m_{b}\left(R_{o} r_{b}\right)^{2}+\frac{1}{2} m_{h}\left(R_{o} r_{h}\right)^{2}, \quad I_{1_{x x}}=\frac{(1-\mu) r_{b}^{2}}{2}+\frac{\mu r_{h}^{2}}{2} \\
\mathrm{I}_{1_{y y}}=\mathrm{I}_{1_{z z}}=\frac{1}{4} m_{b}\left(R_{o} r_{b}\right)^{2}+\frac{1}{2} m_{b}\left(R_{o} h_{b}\right)^{2}+m_{b} R_{o}^{2}(\mu l)^{2} \\
\quad+\frac{1}{4} m_{h}\left(R_{o} r_{h}\right)^{2}+\frac{1}{2} m_{h}\left(R_{o} h_{h}\right)^{2}+m_{h} R_{o}^{2}(l-\mu l)^{2} \\
I_{1_{y y}}=I_{1_{z z}}=\frac{(1-\mu)}{4}\left[r_{b}^{2}+2 h_{b}^{2}+4(\mu l)^{2}\right] \\
+\frac{\mu}{4}\left[r_{h}^{2}+2 h_{h}^{2}+4(l-\mu l)^{2}\right]
\end{gathered}
$$

which only trades the three parameters $I_{1_{x x}}, I_{1_{y y}}$, and $I_{1_{z z}}$ for the parameters $\mu, l$, and the four normalized dimensions $r_{b}, h_{b}, r_{h}$, and $h_{h}$. But we can let these dimensions tend toward zero (because they are comparatively small, given the problem's normalization) and also choose that normalization so that $l$ is always unity. Then the moments of inertia simplify to

$$
I_{1_{x x}} \ll I_{1_{y y}}, \quad I_{1_{y y}}=I_{1_{z z}}=\mu-\mu^{2}
$$

Recall that the system will be fully controllable only if $I_{1_{x x}}$ is not allowed to approach zero, which would lead to an effective rank degeneracy. This, plus the reality of the physical system, is why we have only stated that $I_{1_{x x}}$ is far less than the other two moments of inertia, rather than setting it to exactly zero, consistent with our elimination of the other body-dimension parameters. Within MATLAB, we set $I_{1_{x x}}$ as a nonzero constant a few orders of magnitude smaller than $I_{1 y y}=I_{1_{z z}}$, and this prevents numerical problems.

Now for the ellipsoid pendulum GT representation, also illustrated in Fig. 1, assume that the largest semi-axis $a$ is aligned with the $\hat{\mathbf{i}}$ unit vector of the GT-fixed frame. The potential and its gradients at any arbitrary relative position vector in that frame $(\mathbf{r}=[x, y, z])$ are given by the following [16]:

$$
\begin{gathered}
u=\frac{3}{4} \int_{\lambda}^{\infty}\left(1-\frac{x^{2}}{a^{2}+p}-\frac{y^{2}}{b^{2}+p}-\frac{z^{2}}{c^{2}+p}\right) \frac{\mathrm{d} p}{\Delta(p)} \\
\frac{\partial u}{\partial \mathbf{r}}=\left[\begin{array}{l}
-\frac{3}{2} x \int_{\lambda}^{\infty} \frac{\mathrm{d} p}{\left(a^{2}+p\right) \Delta(p)} \\
-\frac{3}{2} y \int_{\lambda}^{\infty} \frac{\mathrm{d} p}{\left(b^{2}+p\right) \Delta(p)} \\
-\frac{3}{2} z \int_{\lambda}^{\infty} \frac{\mathrm{d} p}{\left(c^{2}+p\right) \Delta(p)}
\end{array}\right]
\end{gathered}
$$

$$
\begin{gathered}
\frac{\partial^{2} u}{\partial \mathbf{r}^{2}}=\operatorname{diag}\left\{\begin{array}{c}
-\frac{3}{2} \int_{\lambda}^{\infty} \frac{\mathrm{d} p}{\left(a^{2}+p\right) \Delta(p)} \\
-\frac{3}{2} \int_{\lambda}^{\infty} \frac{\mathrm{d} p}{\left(b^{2}+p\right) \Delta(p)} \\
-\frac{3}{2} \int_{\lambda}^{\infty} \frac{\mathrm{d} p}{\left(c^{2}+p\right) \Delta(p)}
\end{array}\right\} \\
+\frac{3}{C(x, y, z, \lambda) \Delta(\lambda)}\left[\begin{array}{ccc}
\frac{x^{2}}{\left(a^{2}+\lambda\right)^{2}} & (\mathrm{sym}) & (\mathrm{sym}) \\
\frac{x y}{\left(a^{2}+\lambda\right)\left(b^{2}+\lambda\right)} & \frac{y^{2}}{\left(b^{2}+\lambda\right)^{2}} & (\mathrm{sym}) \\
\frac{x z}{\left(a^{2}+\lambda\right)\left(c^{2}+\lambda\right)} & \frac{y z}{\left(b^{2}+\lambda\right)\left(c^{2}+\lambda\right)} & \frac{z^{2}}{\left(c^{2}+\lambda\right)^{2}}
\end{array}\right]
\end{gathered}
$$

where $p$ is just the constant of integration for the elliptic integrals, and

$$
\begin{gathered}
C(x, y, z, \lambda)=\frac{x^{2}}{\left(a^{2}+\lambda\right)^{2}}+\frac{y^{2}}{\left(b^{2}+\lambda\right)^{2}}+\frac{z^{2}}{\left(c^{2}+\lambda\right)^{2}} \\
\Delta(p) \triangleq \sqrt{\left(a^{2}+p\right)\left(b^{2}+p\right)\left(c^{2}+p\right)}
\end{gathered}
$$

and similarly for $\Delta(\lambda)$. The bound $\lambda$ for the integration is given by the single positive root of

$$
\Phi(\lambda) \triangleq \frac{x^{2}}{a^{2}+\lambda}+\frac{y^{2}}{b^{2}+\lambda}+\frac{z^{2}}{c^{2}+\lambda}-1
$$

In the specific case that we should have for both equilibria, where $x=-r_{d}$ and $y=z=0$ so that $\mathbf{r}_{e q}=\left[-r_{d}, 0,0\right]$, this root is $\lambda=r_{d}^{2}-a^{2}$. The preceding potential and gradients all simplify so that we can easily identify

$$
\begin{gathered}
u_{x}=\epsilon_{a} r_{d}, \quad u_{y y}=-\epsilon_{b}, \quad u_{z z}=-\epsilon_{c} \\
u_{x x}=-\epsilon_{a}+\frac{3}{r_{d} \sqrt{\left(b^{2}-a^{2}+r_{d}^{2}\right)\left(c^{2}-a^{2}+r_{d}^{2}\right)}}
\end{gathered}
$$

Herein, each $\epsilon_{i}$ is shorthand denoting the standard elliptic integral result, with the $i$ semi-axis as a parameter and the preceding integration bound for this case used; that is,

$$
\epsilon_{i}=\frac{3}{2} \int_{r_{d}^{2}-a^{2}}^{\infty} \frac{\mathrm{d} p}{\left(i^{2}+p\right) \Delta(p)}
$$

These elliptic integrals are treated as a black-box function [the same as, for example, $\cos (x)$ ], because general algorithms for their efficient solution are widely available. We also redefine the moments 
of inertia as (given $a>b>c$ and the alignment shown in the figure)

$$
\begin{array}{ll}
\mathrm{I}_{1_{x x}}=\frac{1}{5} m_{1}\left(b^{2}+c^{2}\right), & I_{1_{x x}}=\frac{1}{5}\left(\bar{b}^{2}+\bar{c}^{2}\right) \\
\mathrm{I}_{1_{y y}}=\frac{1}{5} m_{1}\left(a^{2}+c^{2}\right), & I_{1_{y y}}=\frac{1}{5}\left(\bar{a}^{2}+\bar{c}^{2}\right) \\
\mathrm{I}_{1_{z z}}=\frac{1}{5} m_{1}\left(a^{2}+b^{2}\right), & I_{1_{z z}}=\frac{1}{5}\left(\bar{a}^{2}+\bar{b}^{2}\right)
\end{array}
$$

\section{Appendix B: Point-Mass and Ellipsoid Representations of Bar GT with Sphere NEO}

For the three-point-mass bar GT, represent the body and the two outboard thruster nodes of the bar GT as points with masses $m_{b}$ (again, the majority of the GT's total mass $m_{1}$ ) and $m_{n}$. In this case, we redefine the internal mass ratio as $\mu=\left(2 m_{n}\right) /\left(m_{b}+2 m_{n}\right)$ and have the distance from one node to the other as normalized length $l$. Immediately assume a choice of normalization length so that the parameter $l$ is unity. With the alignment of the GT-fixed frame for the bar GT, the key vectors at both desired equilibria are

$$
\begin{aligned}
& \mathbf{r}_{\mathrm{eq}}=\left[\begin{array}{lll}
-r_{d} & 0 & 0
\end{array}\right]^{T}, \quad \boldsymbol{\rho}_{b}=\left[\begin{array}{lll}
0 & 0 & 0
\end{array}\right]^{T} \\
& \boldsymbol{\rho}_{+n}=\left[\begin{array}{lll}
0 & \frac{1}{2} & 0
\end{array}\right]^{T}, \quad \boldsymbol{\rho}_{-n}=\left[\begin{array}{lll}
0 & -\frac{1}{2} & 0
\end{array}\right]^{T}
\end{aligned}
$$

From this, we obtain the basic mutual potential and its gradients and employ the new definition of $\mu$, such that we can identify

$$
\begin{gathered}
x=-r_{d}, \quad u_{x}=\frac{1-\mu}{r_{d}^{2}}+\frac{\mu r_{d}}{\left(r_{d}^{2}+\frac{1}{4}\right)^{\frac{3}{2}}} \\
u_{x x}=\frac{2(1-\mu)}{r_{d}^{3}}+\frac{2 \mu r_{d}^{2}-\frac{\mu}{4}}{\left(r_{d}^{2}+\frac{1}{4}\right)^{\frac{5}{2}}} \quad u_{y y}=\frac{-(1-\mu)}{r_{d}^{3}}+\frac{-\mu r_{d}^{2}+\frac{\mu}{2}}{\left(r_{d}^{2}+\frac{1}{4}\right)^{\frac{5}{2}}} \\
u_{z z}=\frac{-(1-\mu)}{r_{d}^{3}}-\frac{\mu}{\left(r_{d}^{2}+\frac{1}{4}\right)^{\frac{3}{2}}}
\end{gathered}
$$

We can also represent the GT geometry with the uniform-density cylinders and massless links shown in Fig. 2 , giving inertia dyad elements of

$$
\begin{aligned}
\mathrm{I}_{1_{x x}} & =\frac{1}{2} m_{b}\left(R_{o} r_{b}\right)^{2}+2\left[\frac{1}{2} m_{n}\left(R_{o} r_{n}\right)^{2}+\left(\frac{R_{o} l}{2}\right)^{2} m_{n}\right] \\
\mathrm{I}_{1_{y y}} & =\frac{1}{4} m_{b}\left(R_{o} r_{b}\right)^{2}+\frac{1}{2} m_{b}\left(R_{o} h_{b}\right)^{2}+2\left[\frac{1}{4} m_{n}\left(R_{o} r_{n}\right)^{2}\right. \\
& \left.+\frac{1}{2} m_{n}\left(R_{o} h_{n}\right)^{2}\right] \\
\mathrm{I}_{1_{z z}} & =\frac{1}{4} m_{b}\left(R_{o} r_{b}\right)^{2}+\frac{1}{2} m_{b}\left(R_{o} h_{b}\right)^{2}+2\left[\frac{1}{4} m_{n}\left(R_{o} r_{n}\right)^{2}\right. \\
& \left.+\frac{1}{2} m_{n}\left(R_{o} h_{n}\right)^{2}+\left(\frac{R_{o} l}{2}\right)^{2} m_{n}\right]
\end{aligned}
$$

Again letting each of the normalized dimensions $r_{b}, h_{b}, r_{n}$, and $h_{n}$ tend toward zero, given the normalization, and using the mass ratios and simplifying, we find that

$$
I_{1_{x x}}=I_{1_{z z}}=\mu / 4, \quad I_{1_{y y}} \ll I_{1_{x x}}
$$

Of course, this introduces an axisymmetry about the $\hat{\mathbf{j}}$ direction that is not present for this configuration, but this is no worse than assuming axisymmetry about the $\hat{\mathbf{i}}$ direction previously for the pendulum configuration. Again, we state only that $I_{1_{y y}}$ is far less than the other two moments of inertia, rather than setting it exactly to zero for physical realism, and because we must not allow $I_{1_{y y}}$ to approach zero if we are to avoid rank degeneracy and maintain full controllability. In numerical work, we again include $I_{1_{y y}}$ as a nonzero constant of a few orders of magnitude smaller than $I_{1_{x x}}=I_{1_{z z}}$.
Next, consider the ellipsoid bar GT, an ellipsoid that is now oriented as shown in Fig. 2. The largest semi-axis $a$ is realigned with the $\hat{\mathbf{j}}$ unit vector of the GT-fixed frame; also assume that the other two semi-axes are equal $(b=c)$. The force potential and its gradients at any arbitrary relative position vector $\mathbf{r}=[x, y, z]$ in the GT-fixed frame are given by exactly the same equations (A4-A6) and the accompanying definitions for the integration bound, etc., but with all instances of $a$ replaced by $b$ and all instances of $b$ concurrently replaced by $a$. For either of our desired equilibria in which $x=-r_{d}$ and $y=z=0$ and using the $b=c$ assumption, these equations simplify greatly again. Let $\epsilon_{i}$ denote the standard elliptic integral result with the $i$ semi-axis as a parameter, just as before, but note that in this case, the integration bound $\lambda=r_{d}^{2}-b^{2}$ is different. Assuming normalization of the semi-axes along with everything else, and assuming again that the largest semi-axis is the normalization length so that $\bar{a}=1$ and $\bar{b}=\bar{c}$, we can then identify

$$
\begin{gathered}
u_{x}=\epsilon_{\bar{b}} r_{d}, \quad u_{x x}=-\epsilon_{\bar{b}}+\frac{3}{r_{d}^{2} \sqrt{1-\bar{b}^{2}+r_{d}^{2}}} \\
u_{y y}=-\epsilon_{a}, \quad u_{z z}=-\epsilon_{\bar{b}}
\end{gathered}
$$

And we also must redefine the normalized moments of inertia as

$$
I_{1_{x x}}=\frac{1}{5}\left(1+\bar{b}^{2}\right), \quad I_{1_{y y}}=\frac{2}{5} \bar{b}^{2}, \quad I_{1_{z z}}=\frac{1}{5}\left(1+\bar{b}^{2}\right)
$$

\section{Acknowledgments}

E. G. Fahnestock would like to acknowledge the support of the U.S. Air Force Office of Scientific Research (AFOSR). Supercomputers used for some simulations herein were provided by funding from Jet Propulsion Laboratory Institutional Computing and Information Services and the NASA Directorates of Aeronautics Research, Science, Exploration Systems, and Space Operations.

\section{References}

[1] Lu, E. T., and Love, S. G., "Gravitational Tractor for Towing Asteroids," Nature, Vol. 438, Nov. 2005, pp. 177-178. doi: $10.1038 / 438177 \mathrm{a}$

[2] Schweickart, R., Chapman, C., Durda, D., and Hut, P., "Threat Mitigation: The Gravity Tractor," Planetary Defense Resources [online database], http://www.aero.org/conferences/planetarydefense/ documents/wpGT\%20(Schweickart).pdf [retrieved 30 Nov. 2007].

[3] Schweickart, R., Chapman, C., Durda, D., and Hut, P., "Threat Mitigation: The Asteroid Tugboat," Planetary Defense Resources [online database], http://www.aero.org/conferences/planetarydefense/ documents/wpAT\%20(Schweickart).pdf, [retrieved 30 Nov. 2007].

[4] Scheeres, D. J., and Schweickart, R. L., "The Mechanics of Moving Asteroids," 2004 Planetary Defense Conference: Protecting Earth from Asteroids, AIAA Paper 2004-1446, Feb. 2004.

[5] Scheeres, D. J., "Spacecraft at Small NEO," Planetary Defense Resources [online database], http://www.aero.org/conferences/ planetarydefense/documents/wp\%20(Scheeres).pdf $\quad$ [retrieved 30 Nov. 2007].

[6] Scheeres, D. J., "Relative Equilibria for General Gravity Fields in the Sphere-Restricted Full 2-Body Problem," Celestial Mechanics and Dynamical Astronomy, Vol. 94, No. 3, Mar. 2006, pp. 317-349. doi:10.1007/s10569-005-6182-2

[7] Izzo, D., "On the Deflection of Potentially Hazardous Objects," Proceedings of the AAS/AIAA Space Flight Mechanics Meeting, Univelt, San Diego, CA, 2005, pp. 611-622; also American Astronautical Society Paper AAS 05-141,

[8] Scheeres, D. J., Hsiao, F.-Y., and Vinh, N., "Stabilizing Motion Relative to an Unstable Orbit: Applications to Spacecraft Formation Flight," Journal of Guidance, Control, and Dynamics, Vol. 26, No. 1, Jan. 2003, pp. 62-73.

[9] Maciejewski, A. J., "Reduction, Relative Equilibria and Potential in the Two Rigid Bodies Problem," Celestial Mechanics and Dynamical Astronomy, Vol. 63, No. 1, 1995, pp. 1-28. doi:10.1007/BF00691912

[10] Fahnestock, E. G., and Scheeres, D. J., "Simulation of the Full Two Rigid Body Problem Using Polyhedral Mutual Potential and Potential Derivatives Approach," Celestial Mechanics 
and Dynamical Astronomy, Vol. 96, Nos. 3-4, Nov. 2006, pp. 317-339. doi:10.1007/s10569-006-9045-6

[11] Hikida, H., and Mizutani, H., "Tensile Strength of Asteroids Predicted from the Relation of Asteroid Size and Spin Period," Lunar and Planetary Science Conference, Houston, TX, Lunar and Planetary Inst., Paper 1444, 11-15 Mar. 2002; available online at http:// www.lpi.usra.edu/meetings/lpsc2002/pdf/1444.pdf [retrieved 30 Nov. 2007].

[12] "Impact Risk," Near Earth Object Program [online database], http:// neo.jpl.nasa.gov/risks/index.html [retrieved 30 Nov. 2007].

[13] Scheeres, D. J., Benner, L., Ostro, S., Rossi, A., Marzari, F., and Washabaugh, P., "Abrupt Alteration of Asteroid 2004 MN4s Spin State During Its 2029 Earth Flyby," Icarus, Vol. 178, No. 1, Nov. 2005, pp. 281-283.

doi:10.1016/j.icarus.2005.06.002

[14] Smirnov, M. A., "On the Rotation of Asteroid 99942 Apophis," Near Earth Objects, Our Celestial Neighbors: Opportunity and Risk, International Astronomical Union Symposium No. S236Cambridge Univ. Press, Cambridge, England, U.K., Aug. 2006, Paper S236-33.

[15] Fahnestock, E. G., Lee, T., Leok, M., McClamroch, N. H., and Scheeres, D. J., "Polyhedral Potential and Variational Integrator Computation of the Full Two Body Problem," AAS/AIAA Astrodynamics Specialist Conference, Keystone, CO, AIAA Paper 2006-6289, 21-24 Aug. 2006.

[16] Danby, J. M. A., Fundamentals of Celestial Mechanics, 2nd ed., Willmann-Bell, Richmond, VA, 1988, pp. 102-111. 